\title{
Distinct structural and catalytic roles for Zap70 in formation of the immunological synapse in CTL
}

\author{
Misty R Jenkins ${ }^{1 \dagger}$, Jane C Stinchcombe ${ }^{1 \dagger}$, Byron B Au-Yeung ${ }^{2,3,5}$, Yukako Asano", \\ Alex T Ritter ${ }^{1,4}$, Arthur Weiss ${ }^{2,3,5}$, Gillian M Griffiths ${ }^{1 *}$
}

${ }^{1}$ Cambridge Institute for Medical Research, University of Cambridge, Cambridge, United Kingdom; ${ }^{2}$ Department of Medicine, University of California, San Francisco, San Francisco, United States; ${ }^{3}$ Department of Microbiology and Immunology, University of California, San Francisco, San Francisco, United States; ${ }^{4}$ Cell Biology and Metabolism Branch, National Institutes of Health, Bethesda, United States; ${ }^{5}$ Howard Hughes Medical Institue, University of California, San Francisco, San Francisco, United States

*For correspondence: gg305@ cam.ac.uk

tThese authors contributed equally to this work

Competing interests: The authors declare that no competing interests exist.

Funding: See page 18

Received: 31 July 2013 Accepted: 22 January 2014 Published: 04 March 2014

Reviewing editor: Diane Mathis, Harvard Medical School, United States

(c) Copyright Jenkins et al. This article is distributed under the terms of the Creative Commons Attribution License, which permits unrestricted use and redistribution provided that the original author and source are credited.
Abstract T cell receptor (TCR) activation leads to a dramatic reorganisation of both membranes and receptors as the immunological synapse forms. Using a genetic model to rapidly inhibit Zap70 catalytic activity we examined synapse formation between cytotoxic $T$ lymphocytes and their targets. In the absence of Zap70 catalytic activity Vav-1 activation occurs and synapse formation is arrested at a stage with actin and integrin rich interdigitations forming the interface between the two cells. The membranes at the synapse are unable to flatten to provide extended contact, and Lck does not cluster to form the central supramolecular activation cluster (cSMAC). Centrosome polarisation is initiated but aborts before reaching the synapse and the granules do not polarise. Our findings reveal distinct roles for Zap70 as a structural protein regulating integrin-mediated control of actin vs its catalytic activity that regulates TCR-mediated control of actin and membrane remodelling during formation of the immunological synapse.

DOI: 10.7554/eLife.01310.001

\section{Introduction}

T cell receptor (TCR) activation induces a highly structured reorganisation of the receptors at the immunological synapse. The mature synapse forms over several minutes and is segregated into three concentric regions, called the central, peripheral and distal supramolecular activation complexes (SMACs) (reviewed in Jenkins and Griffiths (2010)). The regions can be distinguished by the differential localization of molecules. For instance, Lck and PKC- $\theta$ cluster with the TCR within the central SMAC (cSMAC), while integrins and associated talin are excluded centrally but accumulate at the peripheral SMAC (pSMAC) (Monks et al., 1998) and actin clusters within the distal SMAC (dSMAC). In secretory synapses, formed by cytotoxic T lymphocytes (CTL), secretion occurs into a small secretory cleft next to the cSMAC and within the pSMAC (Stinchcombe et al., 2001b). The membranes around the secretory cleft are very tightly opposed around the area where cytotoxic proteins are secreted. Exactly how this organisation of membranes is set up as CTL and target cells interact is not known.

Zap70 is a key kinase in the TCR signalling pathway. Via its tandem SH2 domains, it associates with tyrosine phosphorylated CD3 and zeta chains and is itself phosphorylated by Src family kinases in response to TCR stimulation (Au-Yeung et al., 2009; van Oers et al., 1996). Zap70 acts as a critical effector of downstream signalling after initial engagement of TCR. Loss of Zap70 in humans leads to Severe Combined Immunodeficiency (SCID) characterized by the absence of CD8 T cells and the presence of non-functional CD4 T cells (Arpaia et al., 1994; Chan et al., 1994; Elder et al., 1994). Defects in 
eLife digest White blood cells are responsible for defending the body against infection and disease. Cytotoxic T-lymphocytes, or cytotoxic T cells, are white blood cells that recognise and kill cells that are infected, cancerous or otherwise damaged. Receptors on the surface of these T cells recognise 'foreign' molecules on the surface of diseased or damaged cells: this activates the T cells, which then release cytotoxic proteins that destroy the target cells.

During this process the T cell and the target cell are brought into close contact with each other, and their membranes undergo a dramatic rearrangement to form an 'immunological synapse'. Although the structure of the immunological synapse is understood in detail, the mechanisms controlling the membrane reorganisation are not well understood. Previous studies have shown that an enzyme called Zap70 needs to be present to activate receptors involved in cell adhesion, termed integrins. Now, Jenkins, Stinchcombe et al. have shown a dual role for Zap70 in the formation of the immunological synapse.

Jenkins, Stinchcombe et al. used mice that had been engineered to produce a modified version of Zap70 that worked as normal until its activity was 'switched off' by the addition of a specific drug. When Zap70 was switched off, integrins were still activated but the formation of the immunological synapse was halted with only finger-tip-like contacts between the T cell and the target cell. These contacts were formed by projections from the T cell made of a protein called actin, which forms a kind of scaffolding within cells. Without active Zap70, the T cell receptors could not trigger the dynamic rearrangement of the actin proteins and the membrane remodelling required to form a tight contact between the two cells. This disrupted the delivery of the cytotoxic proteins to their target. These results clearly show that Zap70 has at least two distinct roles that it must carry out for an immunological synapse to form.

DOI: 10.7554/eLife.01310.002

thymic development are revealed in mice deficient in Zap70 where no mature T cells develop due to a block in positive selection (Negishi et alo, 1995; Kadlecek et alı, 1998). Due to developmental abnormalities, studies on the role of Zap70 in CTL-mediated killing have been limited.

The derivation of mice expressing an engineered Zap70 mutant, the catalytic activity of which can be blocked by the use of a small molecule inhibitor (Levin et al., 2008; Au-Yeung et al., 2010) has changed this. This analog-sensitive Zap70 protein [Zap70(AS)] has a methionine to alanine substitution in its catalytic site which allows it to accommodate the bulky ATP-competitive inhibitor, 3-MB-PP1, which impairs Zap70(AS) catalytic function but has little effect on wild-type Zap70. This model, with Zap70(AS) controlled by the addition of a rapidly acting small molecule inhibitor that is genetically selective, has opened the way to studying the role of Zap70 in functional mature T cells. Importantly, this system is able to distinguish the roles played by the catalytic activity of Zap70 as opposed to its structural contributions since the inhibited kinase is present, associates with the TCR, is tyrosine phosphorylated by Lck and has the capacity to recruit other signalling molecules.

Initial studies with this system have shown that, when added to CD4 T cells containing the Zap70(AS) allele, 3-MB-PP1 inhibits Zap70(AS) catalytic activity within $30 \mathrm{~s}$, thereby leading to loss of LAT and ERK phosphorylation and ablation of the calcium increase in response to TCR cross-linking. Inhibition of Zap70(AS) does not affect its phosphorylation by the upstream kinase, Lck, nor does it affect the catalytic activity of T cells expressing wild-type Zap70. Intriguingly, studies of CD4 T cells revealed that Zap70 catalytic activity was not required for integrin activation, but rather that Zap70 plays a catalyticindependent role in integrin activation (Au-Yeung et al., 2010). The Zap70(AS) model therefore provides an unprecedented opportunity for examining the role of integrin vs TCR mediated events during the formation of the immunological synapse as TCR activation is required for integrin activation (Burbach et al., 2007). This is particularly interesting since integrin activation alone has been shown to trigger polarisation of both centrosome and secretory granules to the immunological synapse in Natural Killer (NK) cells; although both activating receptor and integrin activation are required for degranulation (Bryceson et al., 2005; March and Long, 2011; Bryceson et al., 2009) as well as remodelling of synaptic actin required for cytokine secretion (Brown et al., 2012). Studies on cytokine secretion from CD4 cells suggest that cdc42 may be involved in actin remodelling at the site of secretion (Chemin et al., 2012). 
Centrosomal docking at the plasma membrane is a key step for polarised secretion from CTL, delivering secretory granules along microtubules to the point of centrosomal contact at the synapse where cortical actin is reduced at the site of secretion (Stinchcombe et al., 2006; Stinchcombe and Griffiths, 2007). The importance of centrosomal docking at the plasma membrane is clear in CTL in which Lck expression is conditionally deleted. In Lck-deficient CTL the centrosome only partially migrates towards the synapse and does not reach the plasma membrane; the secretory granules are not delivered to the synapse, and targets are not killed (Tsun et al., 2011).

Previous studies have revealed roles for Lck, LAT, SLP76 and Zap70 (Lowin-Kropf et al., 1998; Kuhne et al., 2003; Tsun et al., 2011) in TCR-induced polarisation of the microtubule organising centre (MTOC) towards the immunological synapse. A study using the Jurkat variant, P116, which lacks Zap70, showed only $50 \%$ of P116 cells were able to polarise their MTOC towards the synapse after superantigen stimulation (which activates via a LAT-independent pathway [Bueno et al., 2006]) while this number increased to $75 \%$ if Zap70 was re-expressed in these cells (Blanchard et al., 2002). This same study showed that cSMAC recruitment of PKC $\theta$ and LAT, was impaired in Zap70-deficient P116 cells, although $\mathrm{CD} 3 \zeta$ clustering was not. The link between these events was not clear.

In this study, we analysed the requirements of Zap70 catalytic activity, distinct from its structural role, in the formation of the immunological synapse, polarisation of the centrosome and granules as well as subsequent cytotoxic functions by effector CD8 CTL. We have taken advantage of the Zap70(AS) system in which the structural protein, Zap70, is present but whose catalytic activity can be rapidly and selectively inhibited. This provided us with a unique system in which to ask whether, as in NK cells, integrin activation is sufficient for centrosome and granule polarisation and whether there are distinct roles for the structural functions vs catalytic activity of Zap70 in formation of the immunological synapse.

\section{Results}

\section{Zap70 catalytic activity is required for CTL mediated killing and cytokine production}

C57BL/6 Zap70(AS) CTL were generated by stimulation with irradiated allogeneic Balb/c splenocytes in vitro, each week for 2 weeks, before using the activated CTL for assays. The level of cytotoxicity was determined by lactate dehydrogenase (LDH) release from P815 target cells. When we examined the ability of Zap70(AS) CTL to induce target cell death in the presence of the 3-MB-PP1 inhibitor, we saw a complete abrogation of killing (Figure 1A). Given that the inhibition of Zap70 has been shown to impair CD4 T cell activation and cytokine production (Au-Yeung et al., 2010), we examined the ability of CTL to produce cytokines after a $5 \mathrm{hr}$ in vitro stimulation with anti-CD3ع. CTL with an inactive Zap70 demonstrated a loss of IFN-y, TNF- $\alpha$ and IL-2 cytokine production (Figure 1B). Therefore, despite being previously activated, CTL still rely on Zap70 signalling for production of cytokines.

\section{Zap70 is essential for organisation of the immunological synapse structure}

We examined the ability of CTL lacking Zap70 catalytic activity, to form immunological synapses. We determined whether they formed cSMAC by looking for the clustering of Lck and PKC- $\theta$ at the synapse and whether a PSMAC was formed by assaying their ability to clear the integrin-associated protein, talin, into a concentric ring around the cSMAC. Zap70-inactive CTL were able to bind and form conjugates with target cells almost as well as Zap70-active CTL, with $60 \%$ of Zap70(AS) CTL $(n=70)$ forming conjugates in the presence of 3-MB-PP1 compared with $67 \%(n=60)$ without inhibitor. When activated Zap70(AS) CTL were conjugated to P815 target cells in the presence of 3-MB-PP1, their ability to clear talin into a ring at the PSMAC was impaired (Figure 2A). Instead, accumulation of talin labelling was seen across the synapse, when viewed in the $z$ plane (Figure 2A, inset). cSMAC formation was also impaired, because the same conjugates displayed a drastic reduction in the accumulation of Lck and PKC- $\theta$ at the cSMAC (Figure 2B,C). These results indicate that Zap70 activity is important in the redistribution of talin and signalling proteins during the formation of a stable synapse.

Previous data have demonstrated that talin is required for F-actin polarisation to the synapse (Wernimont et al., 2011). Therefore, given the loss of talin clearance, immunofluorescence microscopy was used to examine the ability of CTL-P815 conjugates to clear actin and form the distal SMAC (dSMAC) in the absence of ZAP activity (Figure $3 A$ ). Conjugates were scored based on the phenotypes 


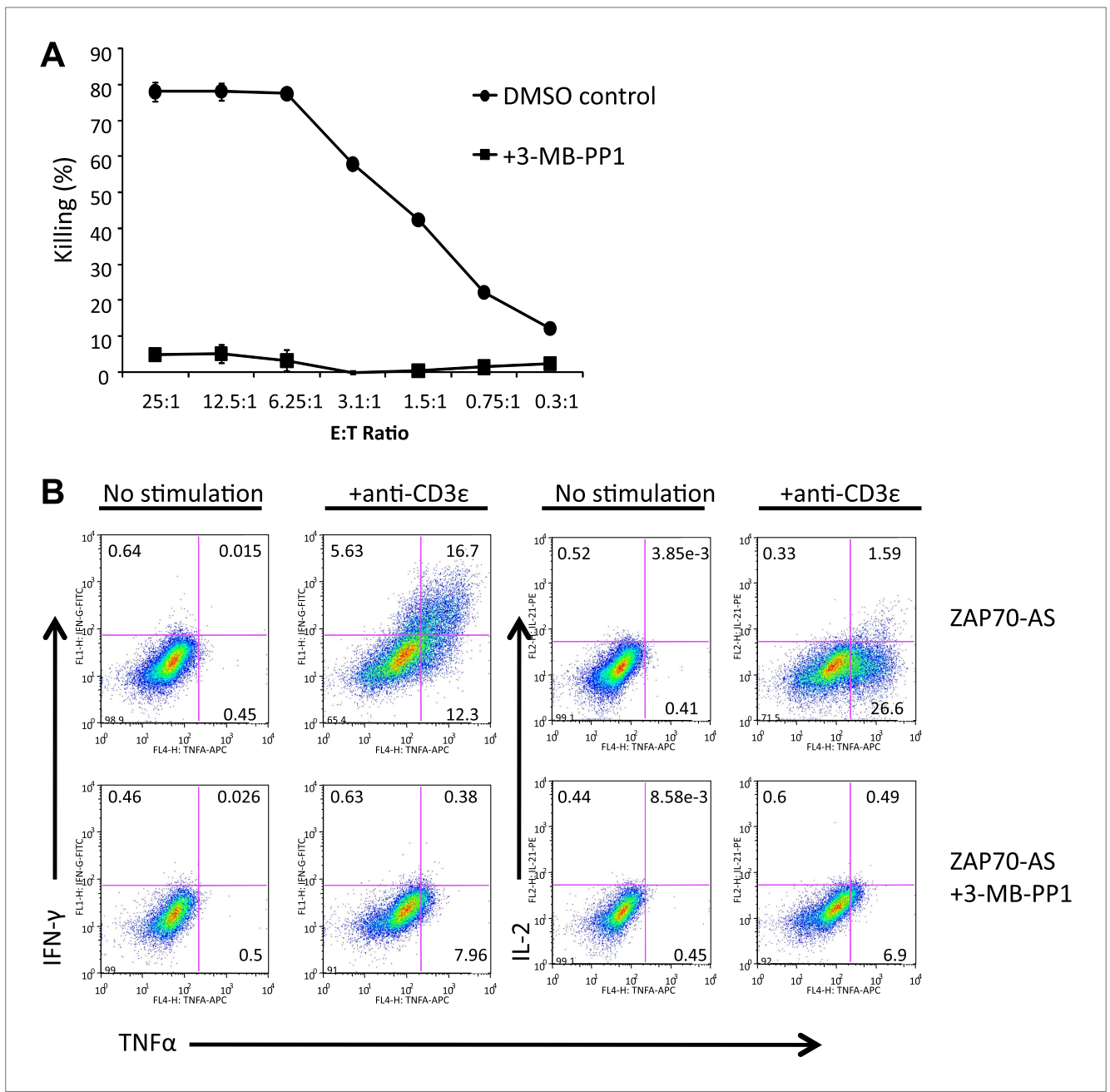

Figure 1. T cell killing and cytokine production is dependent on the catalytic activity of Zap70. (A) Target cell lysis of P815 targets by Zap70(AS) CTL in the presence (squares) or absence (circles) of $10 \mu M$ 3-MB-PP1. Graphs show the mean percentage cytotoxicity of triplicates \pm SD for effector to target $(E: T)$ ratios shown; representative of three independent experiments. (B) FACS analysis of intracellular staining for IFN- $y$ ( $y$-axes) TNF- $\alpha$ and IL2 ( $x$-axes) production by Zap70(AS) CTL stimulated with anti-CD3 $\pm 10 \mu \mathrm{M}$ 3-MB-PP1.

DOI: 10.7554/eLife.01310.003

of total actin clearance, partial, or no clearance from the synapse as described in 'Materials and methods' (Figure 3A). The majority of conjugates formed with Zap70-active CTL accumulate actin at the contact site, which clears into a ring of actin at the dSMAC of the synapse $(76.5 \%, n=65)$ (Figure $3 B$, black) consistent with other studies of CTL (Tsun et al., 2011; Zhao et al., 2012). In contrast, when CTL lack Zap70 catalytic activity, they lose this ability, and the proportion of conjugates displaying clearance drops to $26 \%(n=83)$ (Figure 3B). When actin clearance is examined in conjugates which do not display a cSMAC $(75 \%, n=83)$, the effect is even more pronounced, with $91.5 \%$ of conjugates failing to clear actin. Interestingly, accumulation of actin at the synapse was still observed in the absence of Zap70 activity (Figure 3C). These results reveal that actin accumulates across the synapse in the absence of Zap70 catalytic activity, but fails to clear to form the outer ring or dSMAC. Moreover, cSMAC formation is impaired in the absence of actin clearance.

\section{Zap70 catalytic activity is required for ERK, but not Vav-1 activation}

Previous studies have shown that phosphorylated ERK may play a role in actin reorganisation as ERK co-localises with actin at the immune synapse, is implicated in granule and MTOC polarisation, and is required for CTL degranulation (Robertson et al., 2005; Jenkins et al., 2009). To determine how the inhibition of Zap70 catalytic activity affects downstream signalling including ERK phosphorylation in 

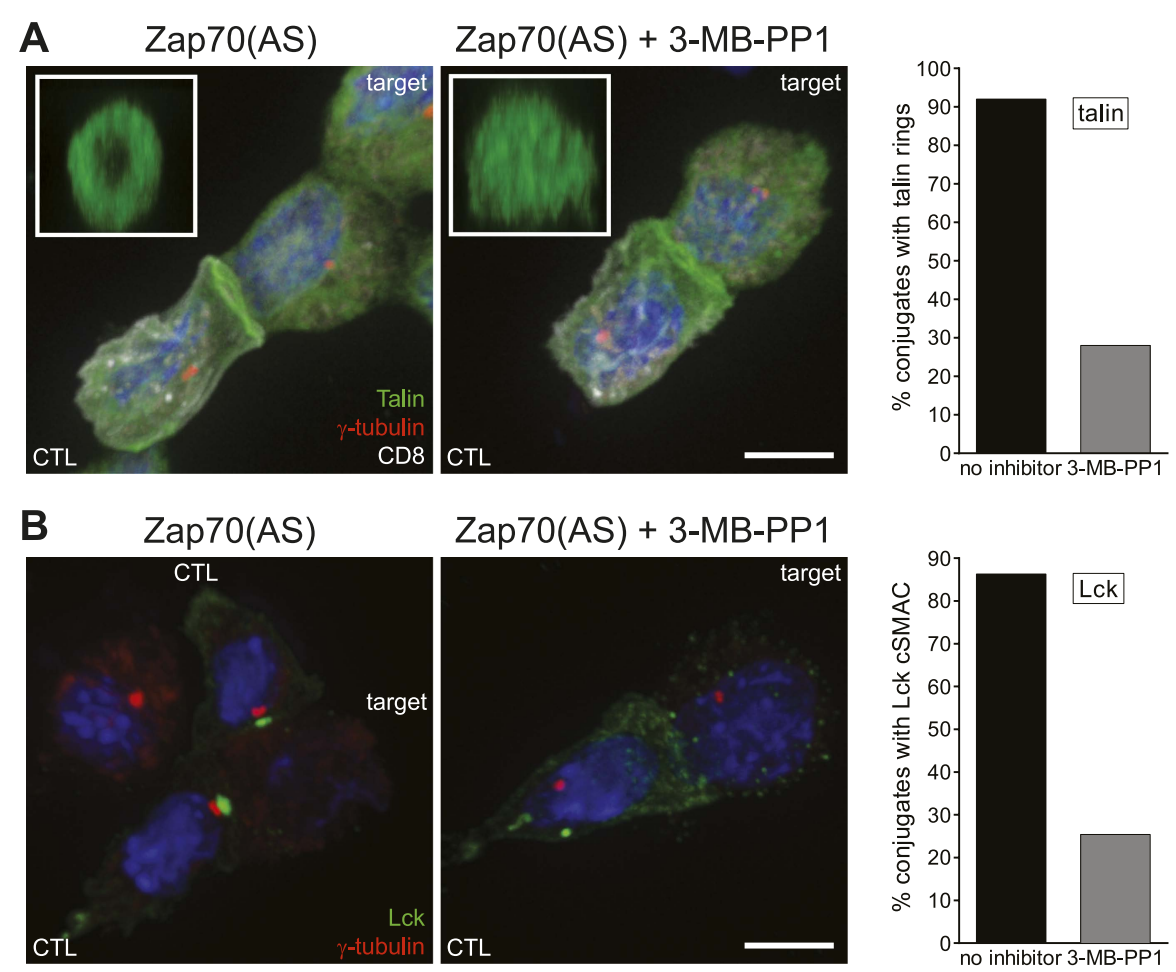

\section{Zap70(AS)}

Zap70(AS) + 3-MB-PP1
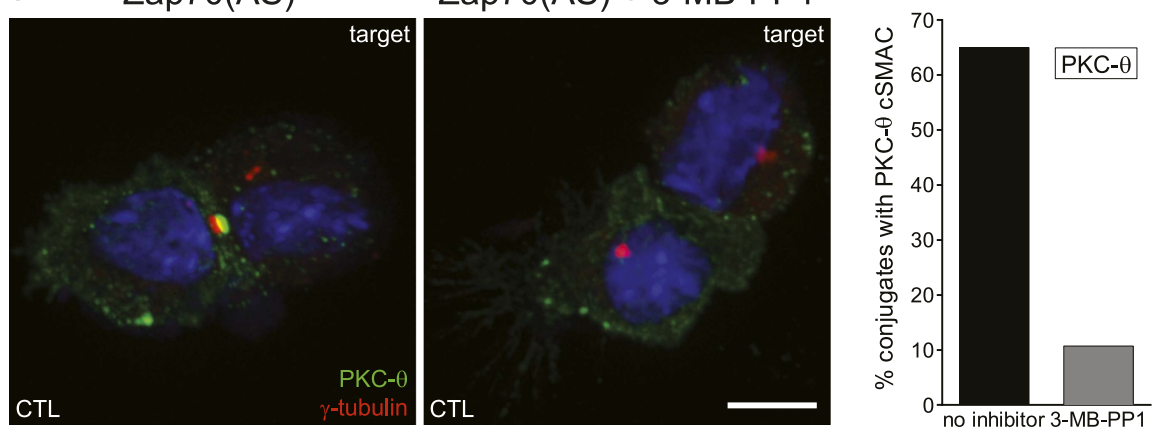

Figure 2. Inhibition of Zap70 activity impairs formation of both the cSMAC and pSMAC. (A-C) Confocal projections of Zap70(AS) CTL conjugated to P815 targets $\pm 10 \mu \mathrm{M}$ 3-MB-PP1. Cells are labelled with Hoechst (nuclei, blue) and antibodies against $\mathrm{Y}$-tubulin (AlexaFluor 546; red) and either talin (A), Lck (B) or PKC- $\theta$ (C) (AlexaFluor-488; green) in the xy plane (scale bar, $5 \mu \mathrm{m}$ ) or as $1 \mu \mathrm{m}$ reconstructions en face across the synapse in the xz plane (insets, scale bar, $3 \mu \mathrm{m})$. Graphs show the quantitation of conjugates with the percentages of conjugates displaying talin rings (A), Lck cSMACs (B) and PKC- $\theta$ cSMACs $(\mathbf{C})$ at the synapse. DOI: 10.7554/eLife.01310.004

CTL, we performed western blot analysis of Zap70+/- and Zap70(AS) CTL lysates (Figure 4A). Inhibition of Zap70 catalytic activity does not impair its capacity to be phosphorylated on tyrosine 319 , which is inducibly phosphorylated upon CD3 crosslinking, and remains similarly phosphorylated in the presence of a high concentration of 3-MB-PP1 $(10 \mu \mathrm{M})$. This result is consistent with Zap70 as a substrate of the upstream Src kinase Lck. However, the phosphorylation of LAT, a substrate of Zap70, as well as PLCY and ERK all depend on Zap70 catalytic activity. These results suggest a signalling pathway from Zap70 via the LAT signalosome and PLCY is required for activation of ERK in CTL. It has also been reported that a PI3K-dependent pathway is required for activating ERK and CTL degranulation (Robertson et al., 2005). To determine whether this pathway may also be dependent on Zap70 activity we probed for phosphorylation of AKT, which is activated downstream of PI3K. 


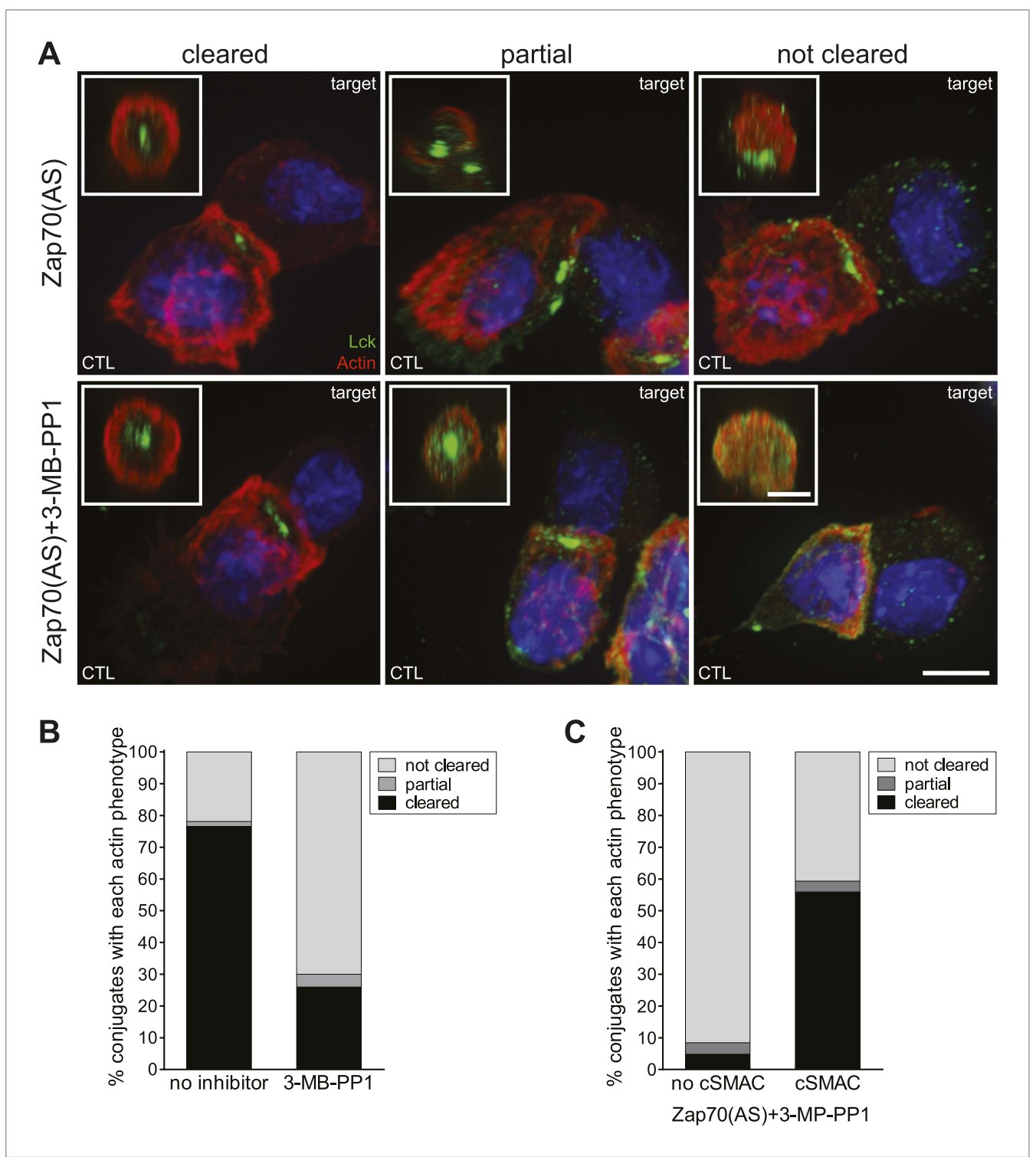

Figure 3. Actin clearance from synapses formed by CTL requires Zap70 activity. (A) Confocal projections of Zap70(AS) CTL conjugated to P815 targets showing actin organisation at the synapse $\pm 10 \mu \mathrm{M} 3-\mathrm{MB}-\mathrm{PP} 1$, in the xy plane (scale bar, $5 \mu \mathrm{m}$ ) or as $1 \mu \mathrm{m}$ reconstructions en face across the synapse in the xz plane (insets, scale bar, $3 \mu \mathrm{m}$ ). Labelling with Hoechst (blue) and antibodies against actin (AlexaFluor-546; red) and Lck (AlexaFluor-488; green). (B) Quantitation of actin organisation at the synapse in conjugates in the presence $(n=83)$ or absence $(n=64)$ of $10 \mu M$ 3-MB-PP1 and (C) for conjugates in the presence of $10 \mu \mathrm{M}$ 3-MB-PP1 $(n=83)$, in which a cSMAC, identified by Lck clustering, is present (cSMAC) or absent (no cSMAC).

DOI: 10.7554/eLife.01310.005

Indeed, Akt phosphorylation was sensitive to Zap70 inhibition, suggesting that ERK is activated downstream of Zap70 through a LAT and PLCY pathway or alternatively through a pathway that also includes AKT.

Since our previous studies had shown that integrin activation occurs in the absence of Zap70 catalytic activity in naïve CD4 T cells (Au-Yeung et al., 2010), we asked whether the integrin-mediated activation of Vav-1 (Gao et al., 2005) was seen in CTL in which Zap70 catalytic activity was inhibited. This was of particular interest because Vav- 1 has been shown to play an important role in both TCRand integrin-mediated activation of actin reorganisation in T cells (del Pozo et al., 2003, GarciaBernal et al., 2009; Tybulewicz, 2005). We found that TCR activation triggers Vav-1 phosphorylation independently of Zap70 kinase activity, with Vav-1 phosphorylation occurring in both Zap70+/- and 


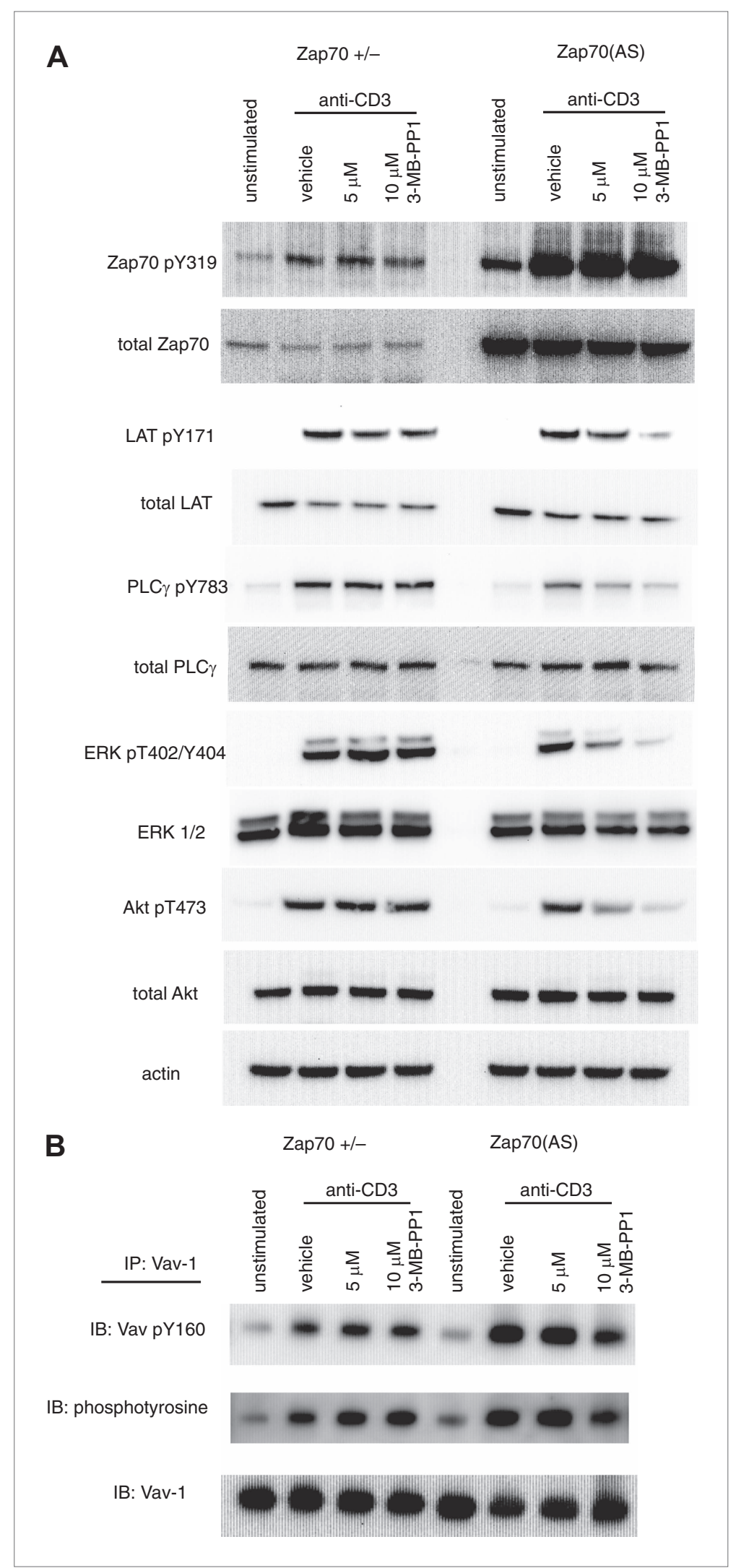

Figure 4. TCR signalling downstream of Zap70 is impaired in the absence of Zap70 catalytic activity. (A) In vitro generated Zap70+/- and Zap70(AS) CTLs were left unstimulated or were stimulated for 2 min by soluble anti-CD3 $(10 \mu \mathrm{g} / \mathrm{ml})$ and cross-linking secondary antibodies, in the presence of vehicle alone (DMSO) or 5 or $10 \mu \mathrm{M}$ 3-MB-PP1. Figure 4. Continued on next page 
Figure 4. Continued

The phosphorylation status of the indicated TCR signalling molecules was determined by Western blot analysis. Molecular weights: ZAP-70, 70kD; LAT, 38kD; PLCY 150kD; ERK 42-44kD; Akt, 60kD; Vav-1, 100kD. (B) Vav-1 immunoprecipitated from Zap70+/- OT-I and Zap70(AS) OT-I CTL treated with vehicle or 3-MB-PP1 as shown. Immunoprecipitates were probed for phosphorylation on tyrosine 160 or total tyrosine phosphorylation of Vav-1. DOI: 10.7554/eLife.01310.006

The following figure supplements are available for figure 4:

Figure supplement 1. ICAM adhesion assays.

DOI: 10.7554/eLife.01310.007

Zap70(AS) CTL lysates from cells treated with 3-MB-PP1, both for overall Vav-1 phosphorylation as well as at $Y 160$, a site that is selectively phosphorylated upon TCR stimulation or av $\beta 3$ integrin-mediated activation (Gao et al., 2005; Miletic et al., 2006). Only a slight decrease in Vav1 phosphorylation is evident in Zap70(AS) CTL with $10 \mu \mathrm{M}$ 3MB-PP1 suggesting Zap70 catalytic function makes only a minimal contribution to Vav-1 phosphorylation (Figure 4B). These results suggest that Vav-1 phosphorylation, and presumably its GEF activation, occurs independently of Zap70 catalytic activity, while the pathway leading to ERK and PI3K activation are dependent on Zap70 catalytic activity. This supports the idea that integrin activation occurs in CTL in which Zap70 is catalytically inhibited, as previously observed in Treg (Au-Yeung et al., 2010).

We examined integrin activation further by asking whether there were differences in adhesion or in the speed of movement of Zap-inhibited CTL. Adherence to an ICAM-1 coated plate was measured before and after TCR activation using anti-CD3E for $10 \mathrm{~min}$. TCR activation increased the percentage of CTL binding to ICAM-1 from $20 \%$ to $30 \%$ for Zap70+/- and from $16 \%$ to $34 \%$ for Zap70 (AS) CTL. 3-MB-PP1 treatment resulted in 32\% binding for Zap70+/- and 25\% for Zap70 (AS) CTL (Figure 4-figure supplement 1). While there does appear to be some reduction in CTL adhesion upon 3-MB-PP1 treatment, these data also suggest there is residual Zap70 catalytic-independent integrin function. We also examined the level of integrin activation by determining the speed of CTL movement using live cell imaging. We found no difference in the speed of movement on an ICAM-1 surface with CTL moving with an average speed of $9 \mu \mathrm{m} / \mathrm{min}$ when Zap70 was catalytically active or inactive (Videos 1 and 2; n>84 each). These results support the idea that Zap70 catalytic activity is not required for integrin activation in CTL. Our finding that conjugate formation is not impaired in Zap70-inactive CTL also supports this model.

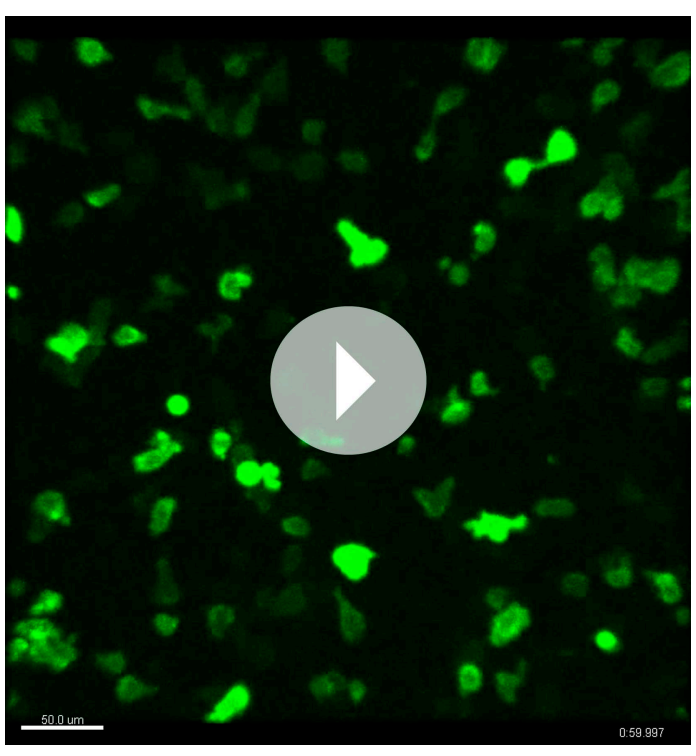

Video 1. Live cell imaging of Zap70(AS) OT-I CTL transfected with Lifeact-EGFP (green) moving on a glass coverslip coated with $0.5 \mu \mathrm{g} / \mathrm{ml} \mathrm{ICAM}-1+0.1 \%$ DMSO. DOI: 10.7554/eLife.01310.011
Thus, distinct TCR-dependent signalling events are influenced by Zap70 scaffolding and kinase activity. Importantly, catalytically inhibited Zap70 can still participate in signal transduction without active catalysis, by interacting with other signalling proteins via its phosphorylated tyrosines. These results in activated CTL are consistent with previous studies in naïve CD4 T cells, which demonstrated a Zap70 kinase-independent scaffold function, where Zap70 forms a complex with the adapter protein Crk, which activates the GTPase Rap1, which subsequently regulates integrinmediated adhesion (Au-Yeung et al., 2010).

\section{Zap70 is essential for centrosome and granule polarisation to the synapse}

Centrosome polarisation to the synapse is an important step in CTL killing, because centrosome docking at the plasma membrane is responsible for directing the cytolytic granules to the secretory cleft at the cSMAC. Given that NK cells can polarise both centrosome and granules to the synapse in response to integrin activation alone 


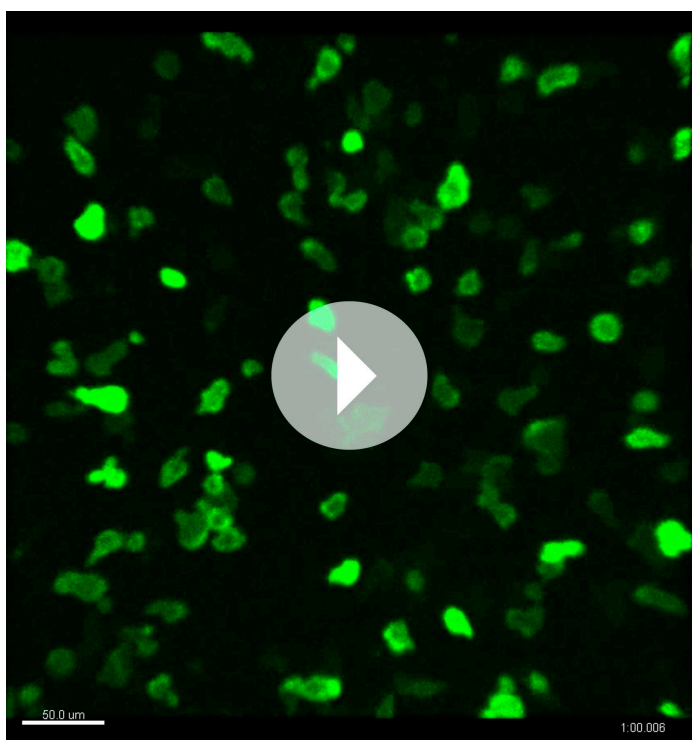

Video 2. Live cell imaging of Zap70(AS) OT-I CTL transfected with Lifeact-EGFP (green) moving on a glass coverslip coated with $0.5 \mu \mathrm{g} / \mathrm{ml} \mathrm{ICAM}-1+10 \mu \mathrm{M}$ 3-MB-PP1

DOI: 10.7554/eLife.01310.012
(Bryceson et al., 2005), it was of interest to ask whether the centrosome could polarise in CTL in which Zap70 was catalytically inhibited but integrin activation could still occur.

Our previous studies have shown that Lck is essential for docking of the centrosome at the plasma membrane since when Lck expression is inducibly turned off in mature CTL, the centrosome polarises towards the immune synapse, but does not dock at the plasma membrane. In the absence of centrosome docking at the plasma membrane, the granules cannot be delivered to the immunological synapse and CTL killing is abolished (Tsun et al., 2011).

Zap70 is a substrate of Lck and its phosphorylation by Lck activates Zap70's kinase activity. Therefore we asked whether centrosome polarisation occurs in the absence of Zap70 catalytic activity by using immunofluorescence microscopy to examine the ability of Zap70(AS) CTL-P815 conjugates to polarise their centrosomes ( $\gamma$-tubulin labelling) (Figure 5A) to the synapse identified by Lck labelling (green) in the presence or absence of inhibitor. Conjugates were classified according to the location of the centrosome relative to the synapse and nucleus (as described in 'Materials and methods' and illustrated in Figure 5A), and by the distance between the centrosome and the synapse (Figure 5B). The majority of conjugates (65\%) with catalytically active Zap70, displayed centrosomes tightly polarised to the cSMAC (Figure 5B) $(n=118)$, in accordance with previously published work (Jenkins et al., 2009). In contrast, Zap70 inhibition disrupted centrosome polarisation, with $54 \%$ of 3-MB-PP1 treated conjugates showing a centrosome location $>5 \mu \mathrm{m}$ from the synapse (Figure $5 B)(n=85)$.

To determine whether inhibition of Zap70 catalytic activity affected granule polarisation, the Zap70(AS) $\pm 3-$ MB-PP1 conjugates were labelled with an antibody to LAMP-1 (CD107a) (Figure 5C, white), a lysosomal membrane protein used as a marker of the secretory granules in $\mathrm{T}$ cells (and lysosomes in the target cells), and scored for the location of the granules, relative to the synapse (as described in 'Materials and methods'). Immunofluorescence microscopy was used to identify granule location relative to the synapse identified by Lck labelling (green) (Figure $5 \mathrm{C}$ ). Although the majority (44\%) of control (Zap70(AS)+DMSO) conjugates $(n=117)$ show granules tightly clustered to the synapse, conjugates treated with 3-MB-PP1 showed a loss of granule polarisation to the synapse (Figure $5 D)(n=92)$. The loss of centrosome and granule polarisation in cells lacking Zap70 catalytic activity is consistent with the loss of cytotoxicity. These results suggest that, in contrast to NK cells, integrin activation is not sufficient for centrosome and granule polarisation to the synapse.

\section{CTL lacking Zap70 catalytic activity initiate but then abort centrosome polarisation}

We also examined centrosome polarisation using live cell microscopy. In order to examine conjugates formed by identical TCR interactions we used CTL derived from lines crossed onto the TCR transgenic OT-I background. Zap70(AS) OT-I CTL were transfected with Lifeact-EGFP (to label polymerised actin) and PACT-mRFP (to label the centrosome) and treated with 3-MB-PP1. Target cell killing by Zap70(AS) OT-I CTL show complete inhibition of killing when treated with 3-MB-PP1 (Figure 6-figure supplement 1). Live cell imaging of Zap70-active OT-I CTL show the centrosome polarising right up to the contact site within 6 min of initial interaction with the target (Figure 6; Video 3; $n=34$ ). However although the centrosome begins to polarise towards the synapse in Zap70(AS) OT-I CTL treated with 3-MB-PP1, the centrosome fails to reach the contact site with the closest point of contact $\sim 3 \mu \mathrm{m}$ at 6 min after initial contact (Figure 6; Video 4; $n=50$ ). In each video examined from Zap-deficient $C T L$, centrosome polarisation began, but aborted before the centrosome docked at the contact site formed by the synapse. 
A

\begin{tabular}{c}
$A$ \\
क \\
\multirow{2}{0}{} \\
0 \\
$\frac{0}{\pi}$ \\
N
\end{tabular}

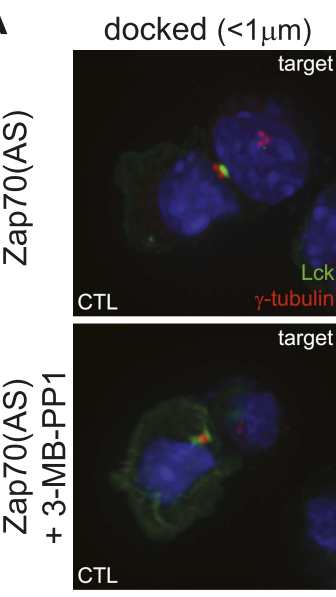

C

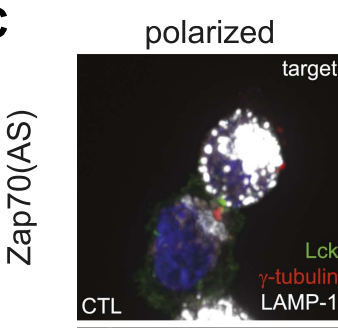

CTL
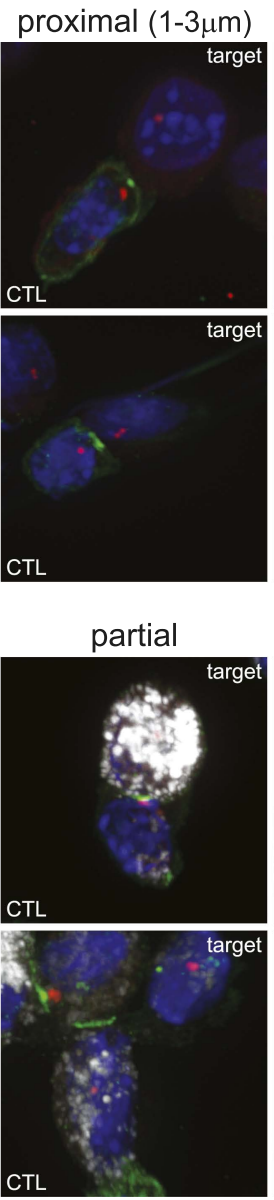

distal $(>3 \mu \mathrm{m})$
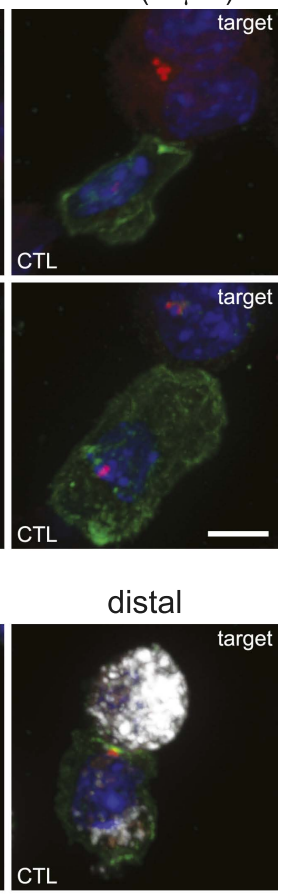

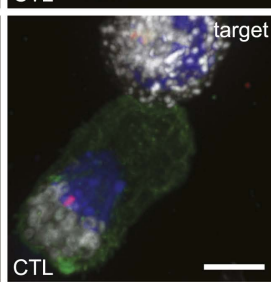

B

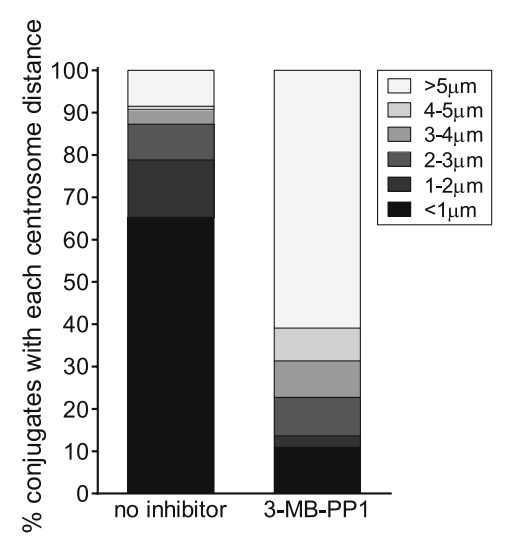

D

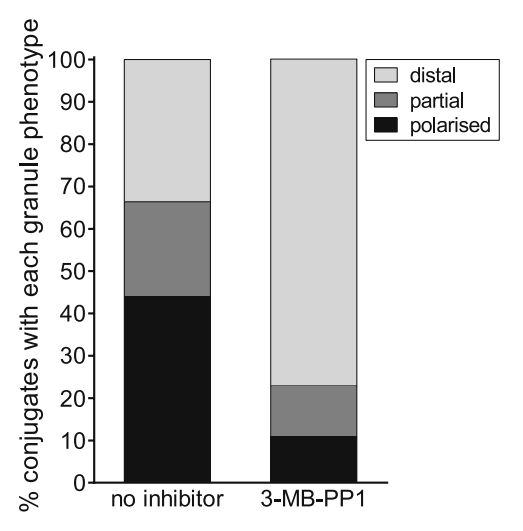

Figure 5. Centrosome and granule polarisation to the synapse is impaired in Zap70 inactive CTL. (A and C) Confocal projections of Zap70(AS) CTL conjugated to P815 targets, labelled with Hoechst (blue) and antibodies against Lck (AlexaFluor-488; green), Y-tubulin (AlexaFluor-546; red) and (C) LAMP-1 (AlexaFluor-633; white) (scale bars, $5 \mu \mathrm{m}$ ) illustrating centrosome (A) and granule (C) polarisation in CTL. Quantitation of conjugates in the presence $(n=325)$ or absence $(n=163)$ of $10 \mu M$ 3-MB-PP1, showing distance of centrosome from the synapse (B) or granule polarisation phenotypes (D), illustrated in (A) and (C), as a percentage of total conjugates formed. (NB LAMP-1 stains both CTL and target lysosomes.)

DOI: 10.7554/eLife.01310.008

\section{Membrane remodelling required for extended contact and secretory cleft formation requires Zap70 catalytic activity}

In order to determine the defect in synapse formation and centrosome polarisation and docking at higher resolution we examined the ultrastructure of the contact site formed between CTL and targets when Zap70 catalytic activity was inhibited. Conjugates formed between CTL and targets were fixed and processed for electron microscopy at 25, 40 and 60 min after mixing.

At 25 (Figure 7A) and 40 (Figure 7C) minutes, the contact sites formed between CTL with active Zap70 and target cells show typical secretory synapses (Stinchcombe et alo, 2001 b) with flat stretches of tight membrane interactions between the cells surrounding a central intercellular gap between the two cell membranes, termed the secretory cleft. The cleft forms an extracellular space containing heterogeneous membranous and granular material. Centrioles, lytic granules and Golgi elements are polarised up to the CTL plasma membrane in the middle of the contact site, opposite the secretory cleft. At these stages of interaction, the target cell appears relatively intact although the endoplasmic reticulum at $40 \mathrm{~min}$ is slightly swollen and vacuolated, characteristic of target cell death (Figure 7C). By $60 \mathrm{~min}$, there is marked evidence of target cell death with extensive endoplasmic reticular vacuolation and swollen mitochondria, with the CTL appearing to retract from an etiolated, dying target (Figure 7E). 

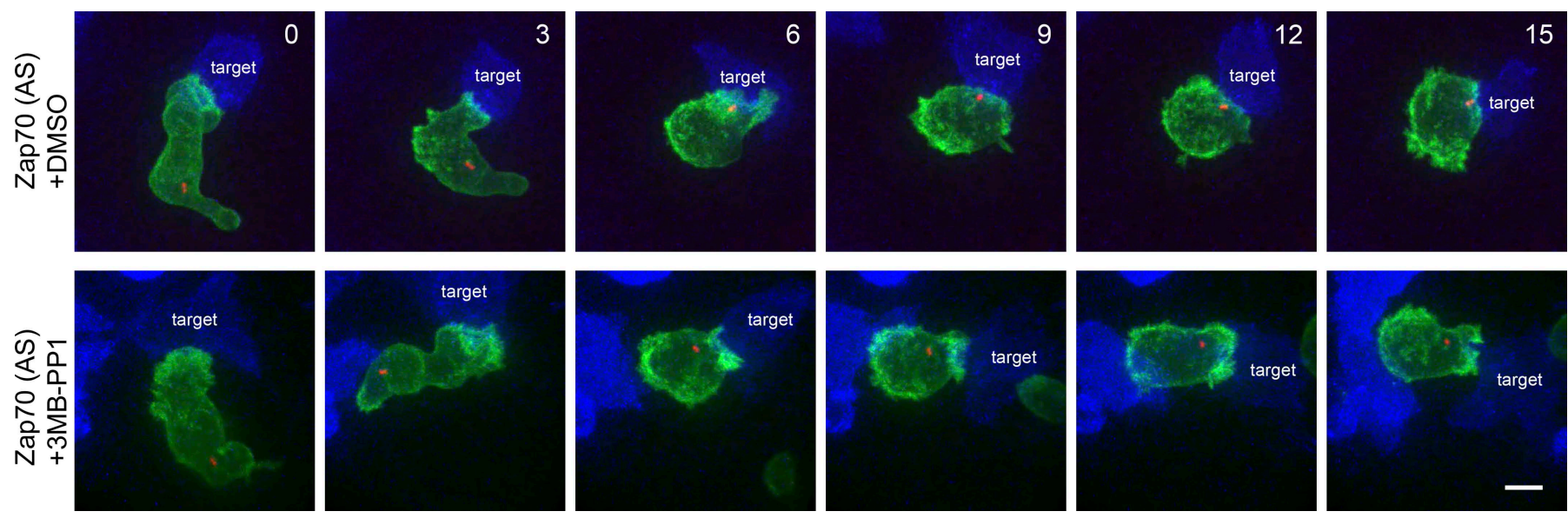

Figure 6. CTL lacking Zap70 catalytic activity show abortive centrosome polarisation. Single frames from Videos 3 and 4 showing 3 min intervals of Zap70(AS) OT-I CTL $\pm 10 \mu \mathrm{M}$ 3-MB-PP1. CTL were transfected with Lifeact-EGFP (green) and mPACT-RFP (centrosome marker, red), and target cells expressing farnesylated mTagBFP2 (blue). Scale bar, $5 \mu \mathrm{m} ; \mathrm{n}=50$ for inhibitor and 34 for control treatments. DOI: 10.7554/eLife.01310.009

The following figure supplements are available for figure 6:

Figure supplement 1. Lysis of targets by Zap70(AS) OT-I CTL is specifically inhibited by 3-MB-PP1.

DOI: 10.7554/eLife.01310.010

In the absence of Zap70 kinase activity the ultrastructure of the synapse is very different (Figure $7 B, D, F)$. There is no evidence of the tight, flat membrane-membrane associations between CTL and target. Secretory clefts are absent, and neither centrioles, Golgi cisternae nor lytic granules are polarised towards the target cells. Instead, the contact site is highly interdigitated, formed of long projections from the CTL surface (black arrowheads). Conjugates formed between CTL lacking Zap70 activity and targets after 25, 40 and 60 min of interaction show similar phenotypes. There is no evidence of target cell death at any of these time points in these samples. Notably points of contact between CTL and target are restricted to the tips of projections, providing limited sites for receptor interactions between the cells.

Quantitation of the EM data reveal that with Zap70(AS) CTL + 3-MB-PP1 the secretory cleft is absent in $>85 \%$ of conjugates analysed at any time point (Figure 7-figure supplement 1A); the centrosome is not docked and instead is found at distances greater than $1 \mu \mathrm{m}$ from the synapse in $>70 \%$ of conjugates (Figure 7-figure supplement 1B) and projections are present between CTL and target (Figure 7-figure supplement 1C). In contrast, in the absence of inhibitor Zap70(AS) CTL showed secretory clefts in $>50 \%$ of conjugates observed at 25 and $40 \mathrm{~min}$, reduced to $27 \%$ only at $60 \mathrm{~min}$, when many of the target cells were dead. Tightly polarised centrosomes are seen most frequently at the 25 min time-point when $69 \%$ of conjugates have at least one centriole $<500 \mathrm{~nm}$ from the plasma membrane and $85 \%$ of conjugates show no projections between CTL and target. Interestingly up to $60 \%$ of CTL lacking Zap70 catalytic activity show the centrosome within $3 \mu \mathrm{m}$ of the synapse in conjugates formed at 25 min (Figure 7-figure supplement 1B), consistent with the initial polarisation up to this point observed in live imaging (Figure 6), decreasing to $35 \%$ in conjugates formed at $60 \mathrm{~min}$.

The unusual ultrastructural morphology of the synapse formed by CTL lacking Zap70 activity most closely resembles that seen in wild-type CTL at very early stages of synapse formation, when the target cell is intact and before the centrosome has polarised to the synapse (Figure 7G). At this stage of synapse formation there are many interdigitations between CTL and target and neither the flattening of the membranes between the two cells, nor the secretory cleft are seen. Here too the contact points between CTL and target are only found at the tips of projections between CTL and target. These observations support the idea that loss of Zap70 catalytic activity arrests the reorganisation of membranes at the synapse at an early stage prior to formation of the secretory cleft.

Importantly these images reveal the ultrastructure that underlies the accumulation of actin seen across the synapses formed by Zap70 catalytically inhibited CTL and at the early stages of synapse 


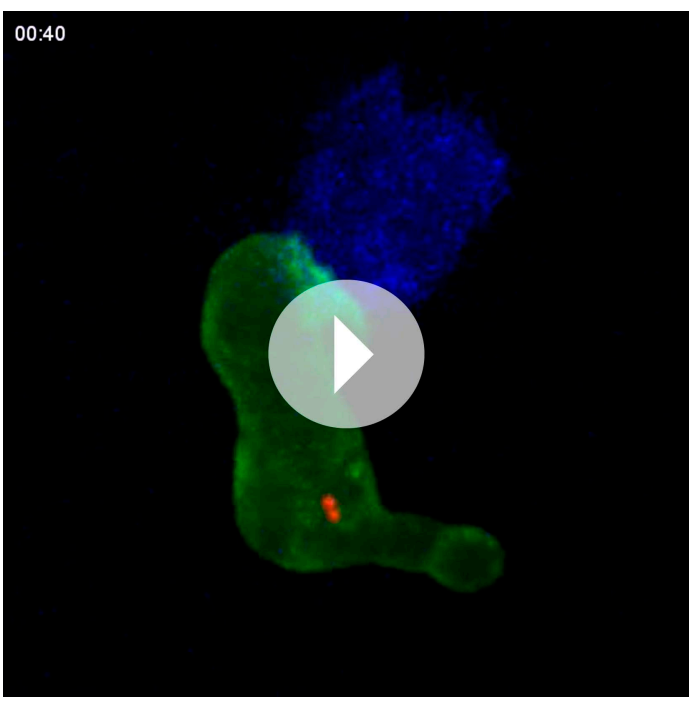

Video 3. Live cell imaging of Zap70(AS) OT-I CTL transfected with Lifeact-EGFP (green) and mPACT-RFP (centrosome marker, red), with EL4 target cells expressing farnesylated mTagBFP2 (blue) +0.1\% DMSO DOI: 10.7554/eLife.01310.013

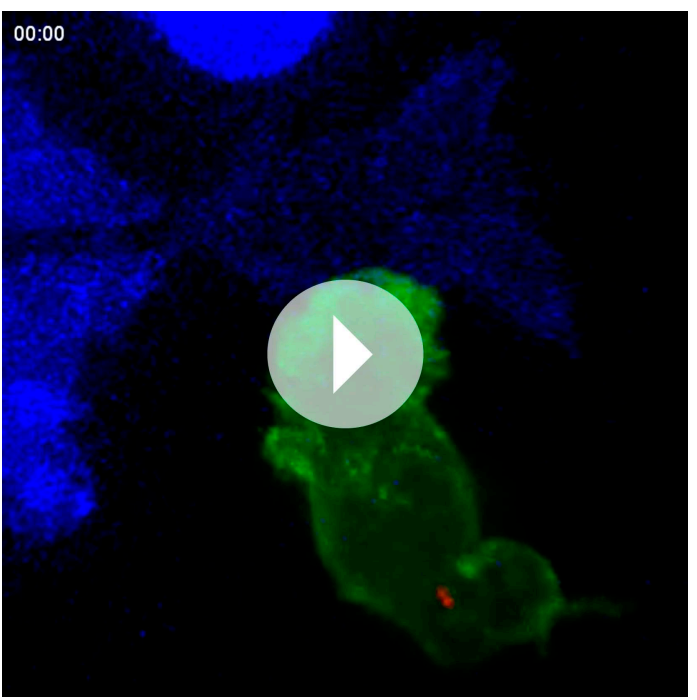

Video 4. Live cell imaging of Zap70(AS) OT-I CTL transfected with Lifeact-EGFP (green) and mPACT-RFP (centrosome marker, red), with target cells labelled with EL4 target cells expressing farnesylated mTagBFP2

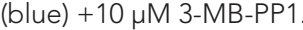

DOI: 10.7554/eLife.01310.014 formation in wild-type CTL, revealing the first link between the clearance of actin and the flattening of the membranes to form an extended area of contact during synapse formation.

\section{Discussion}

In this paper we make use of the Zap70(AS) model to study the role of Zap70 catalytic activity in CTL. While earlier studies revealed that target cell killing was inhibited in the absence of Zap70 activity (Au-Yeung et al., 2010), the underlying mechanisms for the loss of cytotoxicity were not investigated. We find that in the absence of Zap70 catalytic activity formation of the immunological synapse is arrested at a stage where actin-rich interdigitations dominate the interface between the two cells, with actin accumulated across the synapse and TCR unable to coalesce to form a cSMAC. The membranes between CTL and target are unable to flatten to provide an extended area of contact and the secretory cleft does not form. Signalling downstream of Zap70 is disrupted and neither centrosome nor granules polarise. Our studies provide the first insights into the membrane reorganisations that accompany actin remodelling to form the mature immunological synapse.

The Zap70(AS) mouse offers a unique opportunity to examine the role of the catalytic activity of Zap70 in CTL, which can be inhibited rapidly and specifically by addition of the inhibitor 3-MB-PP1. Previous studies using Zap70(AS) CD4 cells demonstrated that although addition of 3-MB-PP1 inhibits phosphorylation of LAT and ERK, Zap70 itself can still be phosphorylated and take part in signalling. We see the same picture in CD8 CTL. Furthermore we show that phosphorylation of PLCY, ERK and AKT are all dependent on Zap70 catalytic activity, suggesting that the LAT signalosome and PLCY are required for ERK activation in $\mathrm{CTL}$, providing a rationale for previous observations implicating both ERK (Robertson et al., 2005) and PLCY (Le Floc'h et al., 2011) in CTL-mediated killing. We also find that catalytically inactive Zap70 can itself be phosphorylated in CTL, supporting a kinase-independent scaffolding role for Zap70 in TCR regulation of integrin-mediated adhesion as reported in Treg cells (Au-Yeung et al., 2010). These results support the idea that the initial stages of synapse formation, during which there is an accumulation of actin across the synapse, may well be integrin mediated. Consistent with this is our finding that Vav-1 phosphorylation mediated by the TCR, and possibly involving integrins (Riteau et al., 2003; Garcia-Bernal et al., 2005, 2009), appears to be independent of Zap70 catalytic activity, with Y160 phosphorylation occurring in Zap70 catalytically inactive CTL. Although earlier studies have implicated Zap70 in the activation of Vav-1 both in vivo (Deckert et al., 1996; Kadlecek et al., 1998; Michel et al., 1998) and in vitro (Brunati et al., 1995; Han et al., 1998), our present study shows phosphorylation of Vav-1 in the absence of Zap-70 catalytic activity, likely mediated by Lck. 


\section{A}

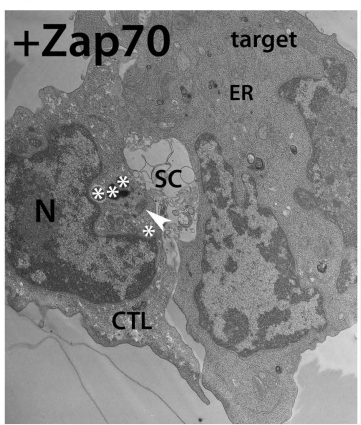

C

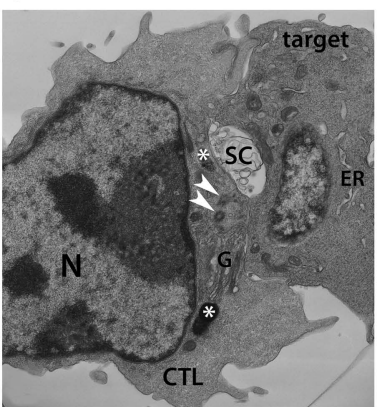

E
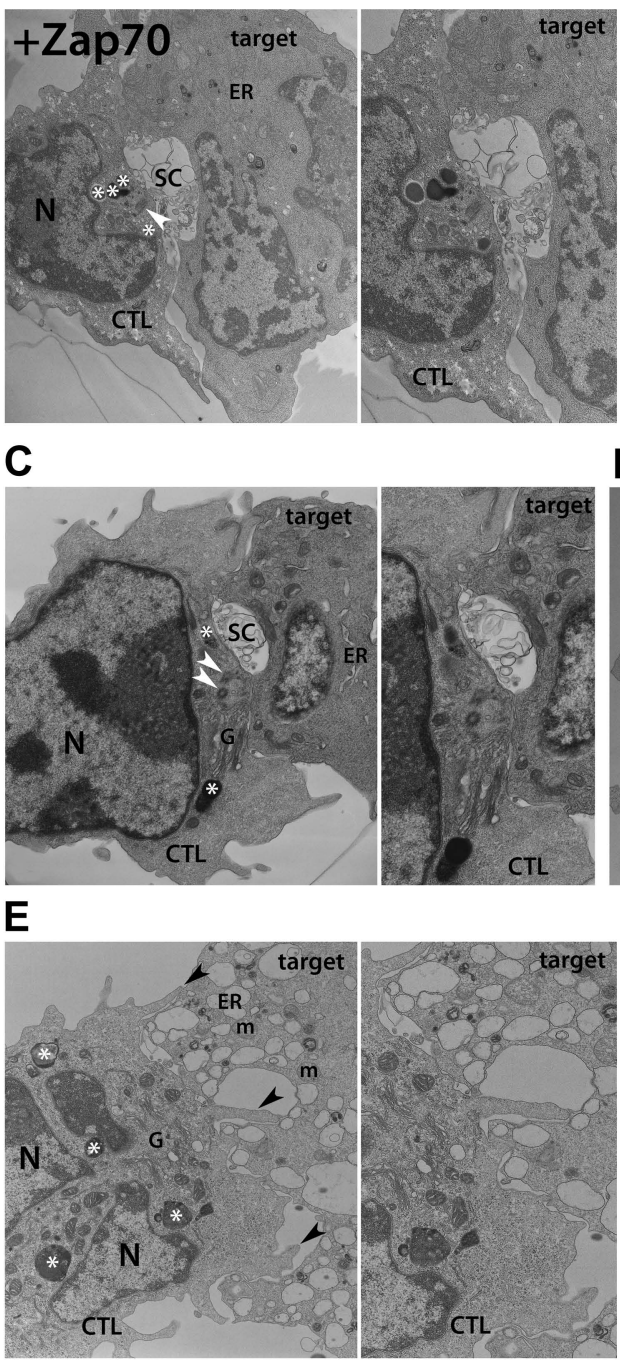

B

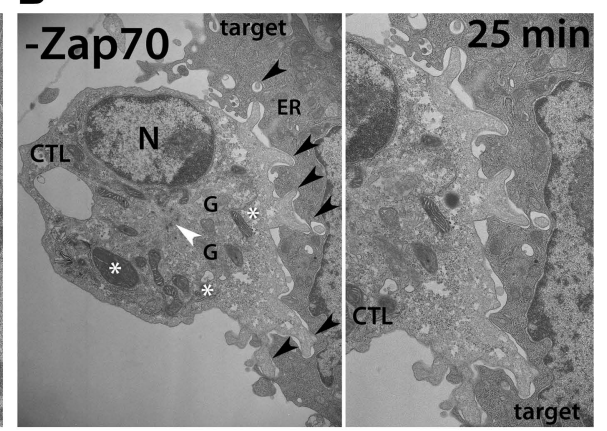

D

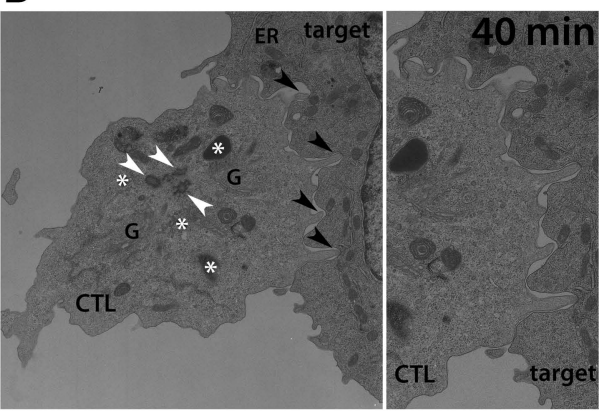

$\mathbf{F}$

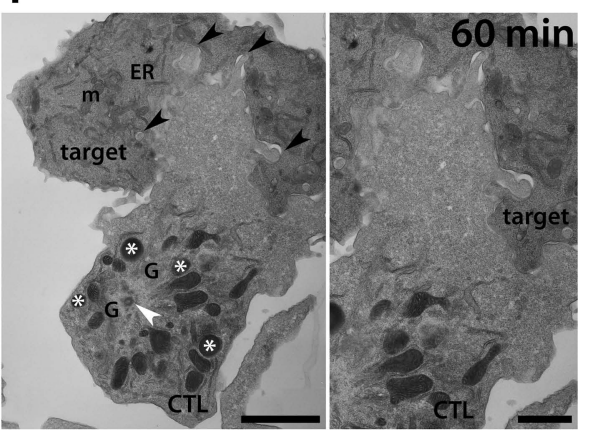

G
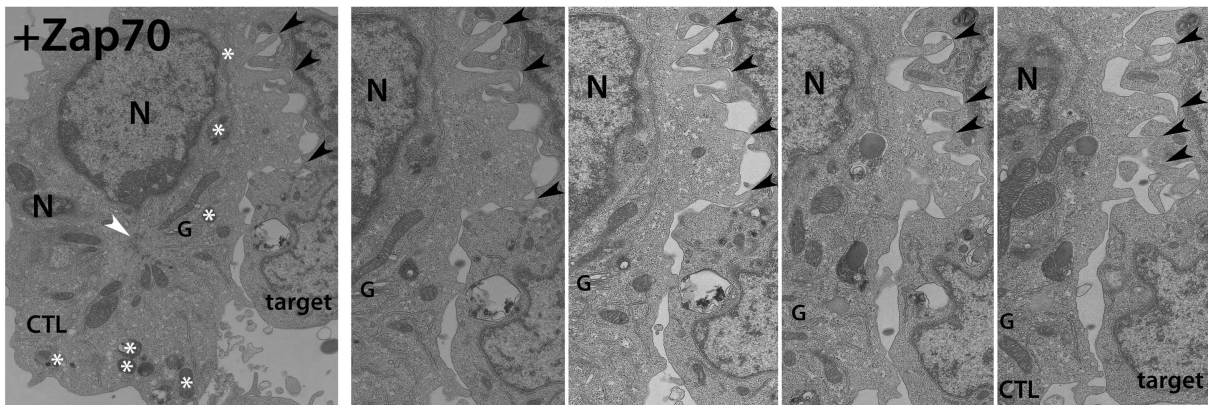

Figure 7. The structure of the immunological synapse is severely impaired in CTL upon Zap70 inhibition. Electron micrographs of single (A-F, left panel $\mathbf{G}$ ) or non-sequential serial (right panels, G) thin (50-70 nm, $\mathbf{A}-\mathbf{E}, \mathbf{G})$ or semi-thin (70-100 nm, F) lead-stained sections through the contact site formed between Zap70(AS) CTL (CTL) and P815 target cells (target), conjugated for 25 (A and B), 40 (C and D) or 60 (E-G) min at 37C in the absence (A, C, E, G) or presence (B, D, F) of $10 \mu \mathrm{M}$ 3-MB-PP1. Secretory cleft (SC); interdigitations, between CTL and targets (black arrowheads); centrioles (white arrowheads), lytic granules (asterisks); Golgi elements (G) and nuclei (N) in CTL, and endoplasmic reticular (ER) and mitochondria $(\mathrm{m})$ in target cells, are indicated in lower power images for $(\mathbf{A}-\mathbf{F})$ and Figure 7. Continued on next page 
Figure 7. Continued

all images for (G). Scale bars: low power images, $2 \mu \mathrm{m}$; high power images bar, $1 \mu \mathrm{m}$. Only the ends of the mother centriole appendages are visible in (B).

DOI: 10.7554/eLife.01310.015

The following figure supplements are available for figure 7:

Figure supplement 1. EM quantitation.

DOI: 10.7554/eLife.01310.016

Our data showing that speed of movement on an ICAM-1 substrate is the same for both Zap70 catalytically active and inactive CTL supports the idea that integrin activation is independent of Zap70 catalytic activity. These results differ from those of an earlier study (Evans et al., 2011) using the less specific inhibitor, piceatannol, where the reduced speed of T cell migration may have resulted from inhibition of target kinases other than Zap70 (http://www.kinase-screen.mrc.ac.uk/screeningcompounds/345911).

The Zap70(AS) model provides a unique opportunity to ask whether, as in NK, integrin activation alone might stimulate centrosome and granule polarisation to the synapse (Bryceson et al., 2005). Our results suggest that CTL differ from NK cells in this respect. Although centrosome polarisation is initiated in CTL lacking Zap70 activity, it is aborted before reaching the synapse, and granule polarisation does not take place. These results suggest an underlying difference in the mechanism of polarisation that may reflect the different roles played by NK and CTL in recognising and destroying virally infected and tumour cells.

In the absence of Zap70 catalytic activity synapse formation is disrupted at both the level of protein reorganisation and ultrastructure. We find that both the integrin-associated talin, as well as actin, accumulate across the immunological synapse, but do not clear to form the concentric pSMAC and dSMAC actin rings characteristic of the synapse. Lck, the kinase that phosphorylates and activates Zap70 and is a marker of the cSMAC, fails to cluster to form a cSMAC. This observation fits with previous observations on P116 Jurkat cells lacking Zap70, which failed to cluster PKC $\theta$ and LAT at the synapse (Blanchard et al., 2002). Our results suggest that the loss of cSMAC formation arises from a failure of actin to clear from the synapse. Actin has been proposed to act as a 'picket fence' (Morone et al., 2006), impeding the movement of proteins laterally within the membrane and an increased density of actin across the synapse might therefore disrupt cSMAC formation by impeding the movement of TCR. In keeping with this model inhibition of actin reorganisation with jasplakinolide on planar lipid bilayers prevents cSMAC formation (Beemiller et al., 2012) as well as with earlier observations that actin dynamics are required for effective TCR signalling stemming either from the use of actin inhibitors (Valitutti et al., 1995; Delon et al., 1998; Tskvitaria-Fuller et al., 2003) or depletion of actin regulatory proteins such as dynamin 2 (Gomez et al., 2005), or ezrin (Roumier et al., 2001), all of which inhibit $T$ cell activation. However our results reveal a much more profound disruption of the whole contact site with actin rich protrusions providing very few points of contact for receptor interactions between the two cells which would impair TCR coalescence to form the cSMAC.

Although it has been known for many years that extensive regions of interdigitations (Kalina and Berke, 1976; Sanderson and Glauert, 1977, 1979), flattened areas and secretory clefts (Bykovskaja et al., 1978; Carpen et al., 1982; Stinchcombe et al., 2001b) can form between killer cells and targets, the functional significance of these differences in membrane organisation has not been clear. Our studies reveal temporal and structural links between the accumulated actin and the extensive interdigitations between CTL and target. Comparing the light and EM images reveals that actin-rich interdigitations seen by EM correspond to confocal images in which actin has accumulated across the synapse, while actin 'clearance' in confocal images corresponds to EM images in which the membranes have flattened between the two cells and in which actin-rich protrusions are only apparent at the edges of the synapse reaching out around the target (Figure 7A,C). Consistent with our observations, Ueda et al noted 'invasive pseudopodia' forming interdigitations between CD4 T cells and B cells at early stages of interaction (Ueda et al., 2011).

Our data reveal that, in the absence of Zap70 catalytic activity, the reorganisation of the membranes between CTL and target does not take place. The secretory cleft is also notably absent from synapses formed by Zap70 catalytically-inactive CTL. Although one possible explanation for loss of the secretory cleft, into which granules secrete their content, could be that this cleft results as a loss of secretion 
from these cells, this seems highly unlikely as the cleft forms properly in synapses made by secretiondeficient CTL lacking Rab27a or Munc13-4 (Stinchcombe et al., 2001a, 2004). The very striking loss of the cleft structure in synapses formed by Zap70 inactive CTL demonstrates that cleft formation requires Zap70 activity.

In the absence of Zap70 activity, downstream TCR signalling is severely impaired, Lck does not cluster to form a cSMAC and although the centrosome begins to polarise it does not reach the synapse. With the loss of centrosome migration, the lytic granules remain dispersed within Zap70 inactive CTL, do not polarise to the synapse and the target cell is not killed. Previous studies with CTL lacking Lck showed that the centrosome was able to polarise around the nucleus towards the synapse, but was unable to dock at the plasma membrane (Tsun et al., 2011). Since live cell imaging was not used to examine centrosome polarisation in Lck-deficient CTL, a direct comparison is not possible. Interestingly actin and talin clearance from the synapse were also impaired in Lck-deficient CTL.

Overall our data support a two-stage model in which the initial accumulation of actin at the synapse is mediated by TCR-facilitated integrin activation (inside out activation of integrins), in which Zap70 plays a scaffolding role (Au-Yeung et al., 2010), but the subsequent reorganisation of actin requires Zap70 catalytic activity. Integrin-mediated actin dynamics proceed normally in Zap-inactive CTL. Not only is Vav-1 phosphorylated, but actin accumulates at the synapse and our live cell studies show that actin reorganisation required for integrin-mediated motility is unimpaired in the absence of Zap70 activity. The loss of Zap70 catalytic activity does not affect the integrin driven accumulation of actin at the synapse, but results in loss of actin reorganisation once the synapse has formed.

Our studies reveal a link between the accumulation of actin and the extensive actin-rich interdigitations formed between CTL and target. It is interesting to note that the only points of contact between CTL and target are at the tips of the interdigitations, and TCR proteins localised to these tips would appear as microclusters. When Zap70 is catalytically active actin clears centrally, the membranes flatten into an extended area of contact and TCR microclusters coalesce to form the CSMAC. Our data are consistent with studies showing that actin clearance is required for the coalescence of microclusters to form the cSMAC (Campi et al., 2005; Babich et alo, 2012; Beemiller et al., 2012; Yi et al., 2012) and we propose that this marks a transition from a highly interdigitated to a flattened interface between the two cells.

In this study we find that inhibition of Zap70 catalytic activity arrests the progress of synapse formation at an early stage. We find that, unlike NK cells (Bryceson et al., 2005), CTL are unable to polarise their centrosome and granules in response to integrin activation alone. Our findings also reveal a surprising new role for Zap70 in controlling the reorganisation of membranes to form the interface at the immunological synapse, including the cSMAC and secretory cleft. Without these rearrangements, the synapse is not functional. Our findings point to distinct roles played by Zap70 as a structural protein regulating integrin-mediated control of actin vs the role of it's catalytic subunit controlling TCR mediated control of actin and membrane remodelling during formation of the immunological synapse.

\section{Materials and methods}

\section{Generation of mouse CTL and cell culture}

Single-cell suspensions of naive splenocytes were generated using a $70 \mu \mathrm{M}$ nylon strainer (Becton Dickinson). Equal numbers of Zap70(AS) or Zap70+/- responders were stimulated with BALB/c-derived stimulator splenocytes (irradiated at 3000 Rad) in RPMI 1640, 10\% FCS, L-glutamine, sodium pyruvate, $50 \mathrm{U} / \mathrm{ml}$ penicillin/streptomycin (Gibco), $50 \mu \mathrm{M} \beta$-2-mercaptoethanol with $100 \mathrm{U} / \mathrm{ml}$ human recombinant IL-2 (Roche, UK) (c-RPMI), and cultured at $37^{\circ} \mathrm{C}$ and $5 \% \mathrm{CO}_{2}$. After 4 days, CTL were purified by separation over Ficoll Histopaque 1083-1 (Sigma-Aldrich, UK), washed three times and resuspended in c-RPMl, at $1 \times 10^{6}$ cells $/ \mathrm{ml}$. CTL were stimulated every 7 days, up to four times. P815 and EL4 mouse target cells were maintained in RPMI, 10\% FCS and L-glutamine. Zap70(AS) OT-I or Zap70+/- OT-I were activated for 4 days with SIINFEKL as previously described (Jenkins et al., 2009).

\section{Reagents, antibodies and western blotting}

3-methylbenzyl-pyrazolopyrimidine (3-MB-PP1) was synthesised as described (Levin et alo, 2008) and a stock solution at $10 \mathrm{mM}(1000 \mathrm{x})$ was dissolved in DMSO. Antibodies used for immunofluorescence studies were: mouse anti-actin (AC-40), rabbit anti-actin and rabbit anti- $\gamma$-tubulin (Sigma-Aldrich, United Kingdom); Mouse anti-mouse Lck (3A5) (Millipore, United Kingdom); rat anti-mouse CD8 (YTS192) (gift from H Waldmann, Oxford University); rat anti-mouse CD107a (LAMP-1, 1BD4) (Developmental 
Studies Hybridoma Bank, University of lowa, lowa City, IA) and all secondary Alexa Fluor antibodies $(405,488,546$ and 633$)$ were obtained from Invitrogen. Western blot analysis of CTL lysates was performed as previously described (Au-Yeung et al., 2010) with antibodies obtained: rabbit antibodies against phospho-ERK T202/Y204, Zap70 Y319, LAT Y171, AKT S473, total LAT and total AKT (Cell Signaling, Danvers, MA); rabbit anti-PLCY Y783 (Biosource, San Diego, CA); anti-phosphotyrosine (4G10), and anti-PLCY (total) (Millipore); anti-ERK 1, ERK 2, and Vav-1 (Santa Cruz, Santa Cruz, CA), Vav Y160 (R\&D Systems, Cambridge, MA) and anti- $\beta$ actin (Sigma, Ronkonkoma, NY). Immunoprecipitations were performed by stimulation of CTL with soluble anti-CD3 $\varepsilon$ and goat anti-Armenian hamster (Jackson Immunoresearch, West Grove, PA) crosslinking antibodies for 2 min, followed by centrifugation and resuspension of cells in 1\% NP-40 alternative lysis buffer with protease inhibitors. Vav-1 was immunoprecipitated with anti-Vav-1 (Santa Cruz) coated protein G sepharose beads (GE Healthcare, Pittsburgh, PA) and eluted from the beads with SDS sample buffer containing $1 \%$ dithiothreitol.

\section{Immunofluorescence and confocal microscopy}

Zap70(AS) CTL (5-8 days after the $3^{\text {rd }}$ or $4^{\text {th }}$ stimulation) and P815 target cells were washed in RPMI, resuspended at $4 \times 10^{6}$ cells $/ \mathrm{ml}$ and mixed 1:1, with $10 \mu \mathrm{M}$ 3-MB-PP1 or DMSO-only control and incubated in suspension for $5 \mathrm{~min}$, before aliquoting onto glass multi-well slides (Hendley), and incubated for a further $15 \mathrm{~min}$ at $37^{\circ} \mathrm{C}$. Samples were then placed on ice and fixed for $5 \mathrm{~min}$ with $-20^{\circ} \mathrm{C}$ methanol, washed six times in PBS and blocked in blocking buffer (PBS +1\% BSA [Sigma]). Primary antibodies were resuspended in PBS $+0.2 \%$ BSA and incubated for $1 \mathrm{hr}$ at room temperature, or overnight at $4^{\circ} \mathrm{C}$. Samples were washed extensively in PBS $+0.2 \%$ BSA before adding secondary antibodies for 40 min at room temperature. Nuclei were stained with Hoechst $(1: 10,000)$ in PBS for 5 min before mounting with 1.5 coverglass and Mowiol. Samples were examined using the Andor Revolution Spinning Disk microscope (with an Olympus microscope, 100x objective) and lasers exciting at 405, 488, 543 and $633 \mathrm{~nm}$.

\section{Live cell imaging}

CTL derived from Zap70(AS) OT-I and Zap70+/- OT-I were nucleofected with PACT-mRFP (Gillingham and Munro, 2000) and Lifeact-EGFP using 5 million cells/nucleofection and the mouse T cell nucleofector kit (Lonza, Germany) with $1 \mathrm{ml}$ mouse T cell nucleofector medium with Component B (Lonza) added post-nuclefection. CTL were topped up with c-RPMI, split between three wells of a 12-well plate and used for imaging $24 \mathrm{hr}$ post nucleofection. EL4 target cells expressing farnesylated mTagBFP2 were pulsed with $1 \mu \mathrm{M}$ SIINFEKL peptide for $40 \mathrm{~min}$ and washed in DMEM to remove peptide and serum, then resuspended in DMEM at $6.5 \times 10^{5} \mathrm{cell} / \mathrm{s} / \mathrm{ml} .250 \mu \mathrm{l}$ of these target cells were aliquoted onto the glass coverslip of $35 \mathrm{~mm}$ glass bottom culture dishes (MatTek, Ashland, MA) pre-coated overnight with $0.5 \mu \mathrm{g} / \mathrm{ml}$ ICAM-1/FC (R\&D Systems, Minneapolis, MN) at $4^{\circ} \mathrm{C}$. Targets were allowed to settle and adhere for $5 \mathrm{~min}$, after which $1.5 \mathrm{ml}$ of RPMl lacking phenol red (Gibco, United Kingdom) supplemented with 10\% FCS, $25 \mathrm{mM} \mathrm{HEPES}$, and $50 \mathrm{U} / \mathrm{ml}$ penicillin and streptomycin (Imaging medium) were added to each chamber with $10 \mu \mathrm{M} 3-\mathrm{MB}-\mathrm{PP} 1$ or $0.1 \%$ DMSO. Approximately 2 million CTL were centrifuged at $1200 \mathrm{rpm}$ for $4 \mathrm{~min}$ and resuspended in $280 \mu$ l of imaging medium with $10 \mu \mathrm{M}$ 3-MB-PP1 or $0.1 \%$ DMSO. CTL were added drop-wise over target cells before imaging was started.

CTL-target interactions were imaged at $37^{\circ} \mathrm{C}$ using an Andor (United Kingdom) Revolution Spinning Disk microscope with $20 x$ or $100 x$ objective, $1.2 x$ camera adapter and environmental chamber (Oko-lab, Japan) for temperature and $\mathrm{CO}_{2}$ regulation. Serial confocal $0.8 \mu \mathrm{m} Z$-stacks were taken at $20 \mathrm{~s}$ intervals with excitation of $405 \mathrm{~nm}, 488 \mathrm{~nm}$, and $561 \mathrm{~nm}$ at each Z plane. Videos were processed and analysed using Andor iQ2 software (Andor Technologies, United Kingdom), Imaris x64 (Bitplane, Switzerland) and Image ( $\mathrm{NIH}, \mathrm{USA})$.

\section{Quantitation of light microscopy}

Conjugate formation was assessed by counting the percentage of CD8 labelled cells conjugated to targets, with all cell nuclei labelled with Hoechst. cSMAC formation was determined by Lck or PKC- $\theta$ clustering at the synapse. Clusters were scored when $>80 \%$ of Lck or PKC- $\theta$ in the cell was clustered in the centre of the synapse. Actin rings were classified as 'cleared' when a contiguous ring of actin formed the dSMAC; any interruption in this ring was scored 'partial' and when a solid wall of actin was observed across the synapse this was scored as 'not cleared' as illustrated in Figure 3. Centrosome position was determined by measuring the distance between the $y$-tubulin labelled centrosome and the Lck or PKC- $\theta$ labelled cSMAC or contact site using Imaris software (Bitplane). Centrosomes 
were categorised as 'docked' when the centrosome was $<1 \mu \mathrm{m}$ from the synapse; proximal when on the synapse side of the nucleus within 1-3 $\mu \mathrm{m}$ and distal when $>3 \mu \mathrm{m}$ from the synapse. Granules were scored as polarised when $>75 \%$ of granules were clustered within $5 \mu \mathrm{m}$ of the synapse; partial when $>50 \%$ of granules were within $5 \mu \mathrm{m}$ of the synapse and distal when $>75 \%$ of granules were $>6 \mu \mathrm{m}$ from the synapse.

\section{Electron microscopy}

CTL were taken 5-6 days after the $2^{\text {nd }}$ or $3^{\text {rd }}$ stimulation and incubated overnight in the presence of $1 \mathrm{mg} / \mathrm{ml}$ horseradish peroxidase, (HRP) (Serva, Germany) added directly to the growth medium, to load the secretory lysosomes via the endocytic pathway. Cells were washed extensively in RPMI to remove serum and residual HRP from the medium, resuspended in RPMI to a final concentration of $1-2 \times 10^{6} \mathrm{cells} / \mathrm{ml}$ and mixed 1:1 with P815 targets (pre-washed and resuspended to $1-2 \times 10^{6} \mathrm{cells} / \mathrm{ml}$ as above) $\pm 10 \mu \mathrm{M}$ 3-MB-PP1. Cells were left in suspension at room temperature for 5 min, after which they were mixed gently (by pipetting up and down), plated in 4-well tissue culture dishes (Nunc) at $0.5 \mathrm{ml} /$ well and incubated at $37^{\circ} \mathrm{C}$ for a further 20,35 , or $55 \mathrm{~min}$ before fixation with $2 \%$ paraformaldehyde and either $1.5 \%$ or $3 \%$ gluteraldehyde (Stinchcombe et al., $2001 \mathrm{~b}$ ). Samples were further processed for DAB cytochemistry, osmium fixation and urynal acetate staining and EPON embedding as previously described (Stinchcombe et al., 2001a, 2001b, 2011; Jenkins et al., 2009). Thin (50-70 nm) and semi-thin (100-150 nm) sections were stained with lead citrate and viewed using a Phillips C100 TEM (FEI). Images were captured using Kodak photographic negative film (Kodak, United Kingdom) and digital electron micrographs produced using a Flextight X5 scanner (Hasselblad, United Kingdom).

\section{Quantitation of EM micrographs}

Thin sections prepared from samples of Zap70(AS) CTL conjugated to targets for 25, 40 or 60 min $\pm 10 \mu \mathrm{M}$ 3-MB-PP1, were stained with lead citrate and imaged using a Phillips C100 TEM microscope. Images of CTL-target conjugates in which at least one centriole was present were analysed, with 22-60 conjugates for each time point $\pm 10 \mu \mathrm{M} 3$-MB-PP1. The secretory cleft was defined as a clear gap within the centre of the contact site between the CTL and target, bounded by an area of tight, flat, membranemembrane contact on each side. Centrosome distance was measured as the shortest distance from the point of the centriole closest to the plasma membrane and the synapse membrane itself. Measurements were classified as $<500 \mathrm{~nm}$ (i.e., one centriole-barrel length, tightly associated), 500-1000 nm (polarised), or >1000 nm (unpolarised) from the plasma membrane. The contact site was defined as the distance between the furthest points of contact between CTL and target at the synapse. The number of membrane extensions projecting from the surface of each CTL with a visible centriole within the contact site was recorded for each CTL condition. Only extensions $>1000 \mathrm{~nm}$ were included in this analysis.

\section{Cytotoxicity and degranulation assays}

Cytotoxicity was examined using the CytoTox 96 Non-Radioactive Cytotoxicity Assay (Promega, United Kingdom). P815 target cells were resuspended in phenol-free RPMI, 2\% FCS at $10^{5} \mathrm{cells} / \mathrm{ml}$ in a round bottom 96-well plate. CTL $\pm 10 \mu \mathrm{M}$ 3-MB-PP1, were added at effector:target (E:T) ratios shown, and plates were incubated at $37^{\circ} \mathrm{C}$ for $4 \mathrm{hr}$. The absorbance of the supernatants at $490 \mathrm{~nm}$ determined the release of lactate dehydrogenase and \% target cell lysis.

\section{Intracellular cytokine staining}

Zap70(AS) CTL were cultured with or without hamster anti-CD3E (145-2C11, Becton Dickinson Bioscience, United Kingdom) for $5 \mathrm{hr}$ at $37^{\circ} \mathrm{C}$ in 96 -well round bottom plates at approx $0.5-2 \times 10^{6}$ cells/well in c-RPMI medium containing $5 \mu \mathrm{g} / \mathrm{ml}$ GolgiPlug (Becton Dickinson) $\pm 10 \mu \mathrm{M}$ 3-MB-PP1. The cells were then washed with PBS (containing $0.1 \%$ BSA and $0.02 \%$ sodium azide), stained with antimouse CD8a-PerCPCy5.5 (Pharmingen) for $30 \mathrm{~min}$ on ice, permeabilised by paraformaldehyde fixation using the BD Cytofix/Cytoperm Kit (Becton Dickinson) and stained for intracellular cytokine production using anti-mouse IFNY-FITC (clone XMG1.2), anti-mouse TNFa (clone MP6-XT22), and antimouse IL-2 (clone JES6-5H4) (Pharmingen, United Kingdom). Lymphocytes were washed and analysed on a FACScalibur and analysed using CellQuestPro software (Becton Dickinson). In each assay, any cytokine positive cells isolated from wells with no were subtracted from the \% cytokine positive cells incubated with peptide to yield the final value. 


\section{ICAM adhesion assay}

CTL (5 $\times 10^{5}$ per well) were stimulated in triplicate wells for $10 \mathrm{~min}$ with $5 \mu \mathrm{g} / \mathrm{ml}$ soluble anti-CD3e (clone 145-2C11) and $50 \mu \mathrm{g} / \mathrm{ml}$ crosslinking goat anti-Armenian hamster (Jackson Immunoresearch) Abs in 96 well plates coated with $10 \mu \mathrm{g} / \mathrm{ml}$ recombinant ICAM-1-Fc (R\&D Systems). Cells were washed three times with pre-warmed media, removed with cell dissociation buffer (Gibco) and plate-bound cells were counted by FACS. The percentage of cells bound was calculated as ([number of live CD4 cells bound to the plate) $\div$ (input number of cells per well]) $\times 100$.

\section{Measurement of cell speed}

Zap70(AS) OT-I and Zap70+/- OT-I CTL were nucleofected with LifeAct-eGFP and imaged as described in 'Live cell imaging' above using the 1.2x camera adapter x20 objective lens. Serial confocal $1.6 \mu \mathrm{m}$ 10 planes of Z-stacks were taken at $20 \mathrm{~s}$ intervals for $15 \mathrm{~min}$ (= 45 time points) with excitation of 488 $\mathrm{nm}$. Videos were analysed using the 'Spots module' of Imaris software (7.6.0 Bitplane) to detect each cell as a spot. Only cells with track durations $>140 \mathrm{~s}$ and track displacement lengths $>13 \mu \mathrm{m}$ were analysed in order to exclude immobile cells. The mean speed for each set of conditions was calculated from 40-50 cells per condition.

\section{Acknowledgements}

We thank Chao Zhang and Kevan Shokat for synthesizing and providing 3-MB-PP1, and Winnie Lui-Roberts for assistance with preparing the figures.

\section{Additional information}

Funding

\begin{tabular}{lll} 
Funder & Grant reference number & Author \\
\hline Wellcome Trust & 075880,100140 & Gillian M Griffiths \\
\hline $\begin{array}{l}\text { Howard Hughes Medical Institute } \\
\text { Australian National Health } \\
\begin{array}{l}\text { and Medical Research Council } \\
\text { (NHMRC) Biomedical Fellowship }\end{array}\end{array}$ & 567082 & Arthur Weiss \\
\hline $\begin{array}{ll}\text { Arthritis Foundation } \\
\begin{array}{l}\text { NIAMS, National Institutes } \\
\text { of Health }\end{array}\end{array}$ & R476 & Misty R Jenkins \\
\hline
\end{tabular}

The funders had no role in study design, data collection and interpretation, or the decision to submit the work for publication.

Author contributions

MRJ, JCS, BBA-Y, YA, ATR, Acquisition of data, Analysis and interpretation of data, Drafting or revising the article; AW, GMG, Conception and design, Analysis and interpretation of data, Drafting or revising the article

\section{References}

Arpaia E, Shahar M, Dadi H, Cohen A, Roifman CM. 1994. Defective T cell receptor signaling and CD8+ thymic selection in humans lacking zap-70 kinase. Cell 76:947-958. doi: 10.1016/0092-8674(94)90368-9.

Au-Yeung BB, Deindl S, Hsu LY, Palacios EH, Levin SE, Kuriyan J, Weiss A. 2009. The structure, regulation, and function of ZAP-70. Immunological Reviews 228:41-57. doi: 10.1111/j.1600-065X.2008.00753.x.

Au-Yeung BB, Levin SE, Zhang C, Hsu LY, Cheng DA, Killeen N, Shokat KM, Weiss A. 2010. A genetically selective inhibitor demonstrates a function for the kinase Zap70 in regulatory $T$ cells independent of its catalytic activity. Nature Immunology 11:1085-1092. doi: 10.1038/ni.1955.

Babich A, Li S, O'connor RS, Milone MC, Freedman BD, Burkhardt JK. 2012. F-actin polymerization and retrograde flow drive sustained PLCY1 signaling during T cell activation. The Journal of Cell Biology 197:775-787. doi: 10.1083/ jcb.201201018.

Beemiller P, Jacobelli J, Krummel MF. 2012. Integration of the movement of signaling microclusters with cellular motility in immunological synapses. Nature Immunology 13:787-795. doi: 10.1038/ni.2364. 
Blanchard N, Di Bartolo V, Hivroz C. 2002. In the immune synapse, ZAP-70 controls T cell polarization and recruitment of signaling proteins but not formation of the synaptic pattern. Immunity 17:389-399. doi: 10.1016/ S1074-7613(02)00421-1.

Brown AC, Dobbie IM, Alakoskela JM, Davis I, Davis DM. 2012. Super-resolution imaging of remodeled synaptic actin reveals different synergies between NK cell receptors and integrins. Blood 120:3729-3740. doi: 10.1182/ blood-2012-05-429977.

Brunati AM, Donella-Deana A, Ruzzene M, Marin O, Pinna LA. 1995. Site specificity of p72syk protein tyrosine kinase: efficient phosphorylation of motifs recognized by Src homology 2 domains of the Src family. FEBS Letters 367:149-152. doi: 10.1016/0014-5793(95)00555-N.

Bryceson YT, Ljunggren HG, Long EO. 2009. Minimal requirement for induction of natural cytotoxicity and intersection of activation signals by inhibitory receptors. Blood 114:2657-2666. doi: 10.1182/ blood-2009-01-201632.

Bryceson YT, March ME, Barber DF, Ljunggren HG, Long EO. 2005. Cytolytic granule polarization and degranulation controlled by different receptors in resting NK cells. The Journal of Experimental Medicine 202:1001-1012. doi: 10.1084/jem.20051143.

Bueno C, Lemke CD, Criado G, Baroja ML, Ferguson SS, Rahman AK, Tsoukas CD, Mccormick JK, Madrenas J. 2006. Bacterial superantigens bypass Lck-dependent T cell receptor signaling by activating a Galpha11-dependent, PLC-beta-mediated pathway. Immunity 25:67-78. doi: 10.1016/j.immuni.2006.04.012.

Burbach BJ, Medeiros RB, Mueller KL, Shimizu Y. 2007. T-cell receptor signaling to integrins. Immunological Reviews 218:65-81. doi: 10.1111/j.1600-065X.2007.00527.x.

Bykovskaja SN, Rytenko AN, Rauschenbach MO, Bykovsky AF. 1978. Ultrastructural alteration of cytolytic T lymphocytes following their interaction with target cells. II. Morphogenesis of secretory granules and intracellular vacuoles. Cellular Immunology 40:175-185. doi: 10.1016/0008-8749(78)90325-8.

Campi G, Varma R, Dustin ML. 2005. Actin and agonist MHC-peptide complex-dependent T cell receptor microclusters as scaffolds for signaling. The Journal of Experimental Medicine 202:1031-1036. doi: 10.1084/ jem.20051182.

Carpen O, Virtanen I, Saksela E. 1982. Ultrastructure of human natural killer cells: nature of the cytolytic contacts in relation to cellular secretion. Journal of Immunology 128:2691-2697.

Chan AC, Kadlecek TA, Elder ME, Filipovich AH, Kuo WL, Iwashima M, Parslow TG, Weiss A. 1994. ZAP-70 deficiency in an autosomal recessive form of severe combined immunodeficiency. Science 264:1599-1601. doi: 10.1126/science.8202713.

Chemin K, Bohineust A, Dogniaux S, Tourret M, Guegan S, Miro F, Hivroz C. 2012. Cytokine secretion by CD4+ T cells at the immunological synapse requires $\mathrm{Cdc42-dependent} \mathrm{local} \mathrm{actin} \mathrm{remodeling} \mathrm{but} \mathrm{not} \mathrm{microtubule}$ organizing center polarity. Journal of Immunology 189:2159-2168. doi: 10.4049/jimmunol.1200156.

Deckert M, Tartare-Deckert S, Couture C, Mustelin T, Altman A. 1996. Functional and physical interactions of Syk family kinases with the Vav proto-oncogene product. Immunity 5:591-604. doi: 10.1016/ S1074-7613(00)80273-3.

del Pozo MA, Schwartz MA, Hu J, Kiosses WB, Altman A, Villalba M. 2003. Guanine exchange-dependent and -independent effects of Vav1 on integrin-induced T cell spreading. Journal of Immunology 170:41-47.

Delon J, Bercovici N, Liblau R, Trautmann A. 1998. Imaging antigen recognition by naive CD4+ T cells: compulsory cytoskeletal alterations for the triggering of an intracellular calcium response. European Journal of Immunology 28:716-729. doi: 10.1002/(SICI)1521-4141(199802)28:02<716::AID-IMMU716>3.0.CO;2-E.

Elder ME, Lin D, Clever J, Chan AC, Hope TJ, Weiss A, Parslow TG. 1994. Human severe combined immunodeficiency due to a defect in ZAP-70, a T cell tyrosine kinase. Science 264:1596-1599. doi: 10.1126/ science.8202712.

Evans R, Lellouch AC, Svensson L, Mcdowall A, Hogg N. 2011. The integrin LFA-1 signals through ZAP-70 to regulate expression of high-affinity LFA-1 on T lymphocytes. Blood 117:3331-3342. doi: 10.1182/ blood-2010-06-289140.

Gao C, Schaefer E, Lakkis M, Blystone SD. 2005. Beta3 tyrosine phosphorylation and alphavbeta3-mediated adhesion are required for Vav1 association and Rho activation in leukocytes. The Journal of Biological Chemistry 280:15422-15429. doi: 10.1074/jbc.M414457200.

Garcia-Bernal D, Parmo-Cabanas M, Dios-Esponera A, Samaniego R, Hernan PDLOD, Teixido J. 2009. Chemokine-induced Zap70 kinase-mediated dissociation of the Vav1-talin complex activates alpha4beta1 integrin for T cell adhesion. Immunity 31:953-964. doi: 10.1016/j.immuni.2009.09.021.

Garcia-Bernal D, Wright N, Sotillo-Mallo E, Nombela-Arrieta C, Stein JV, Bustelo XR, Teixido J. 2005. Vav1 and Rac control chemokine-promoted T lymphocyte adhesion mediated by the integrin alpha4beta1. Molecular Biology of the Cell 16:3223-3235. doi: 10.1091/mbc.E04-12-1049.

Gillingham AK, Munro S. 2000. The PACT domain, a conserved centrosomal targeting motif in the coiled-coil proteins AKAP450 and pericentrin. EMBO Reports 1:524-529. doi: 10.1093/embo-reports/kvd105.

Gomez TS, Hamann MJ, Mccarney S, Savoy DN, Lubking CM, Heldebrant MP, Labno CM, Mckean DJ, Mcniven MA Burkhardt JK, Billadeau DD. 2005. Dynamin 2 regulates T cell activation by controlling actin polymerization at the immunological synapse. Nature Immunology 6:261-270. doi: 10.1038/ni1168.

Han J, Luby-Phelps K, Das B, Shu X, Xia Y, Mosteller RD, Krishna UM, Falck JR, White MA, Broek D. 1998. Role of substrates and products of PI 3-kinase in regulating activation of Rac-related guanosine triphosphatases by Vav. Science 279:558-560. doi: 10.1126/science.279.5350.558.

Jenkins MR, Griffiths GM. 2010. The synapse and cytolytic machinery of cytotoxic T cells. Current Opinion In Immunology 22:308-313. doi: 10.1016/j.coi.2010.02.008. 
Jenkins MR, Tsun A, Stinchcombe JC, Griffiths GM. 2009. The strength of T cell receptor signal controls the polarization of cytotoxic machinery to the immunological synapse. Immunity 31:621-631. doi: 10.1016/j. immuni.2009.08.024.

Kadlecek TA, Van Oers NS, Lefrancois L, Olson S, Finlay D, Chu DH, Connolly K, Killeen N, Weiss A. 1998. Differential requirements for ZAP-70 in TCR signaling and T cell development. Journal of Immunology 161:4688-4694.

Kalina M, Berke G. 1976. Contact regions of cytotoxic T lymphocyte-target cell conjugates. Cellular Immunology 25:41-51. doi: 10.1016/0008-8749(76)90095-2.

Kuhne MR, Lin J, Yablonski D, Mollenauer MN, Ehrlich LI, Huppa J, Davis MM, Weiss A. 2003. Linker for activation of T cells, zeta-associated protein-70, and Src homology 2 domain-containing leukocyte protein-76 are required for TCR-induced microtubule-organizing center polarization. Journal of Immunology 171:860-866.

Le Floc'h A, Jalil A, Franciszkiewicz K, Validire P, Vergnon I, Mami-Chouaib F. 2011. Minimal engagement of CD103 on cytotoxic $\mathrm{T}$ lymphocytes with an E-cadherin-Fc molecule triggers lytic granule polarization via a phospholipase Cgamma-dependent pathway. Cancer Research 71:328-338. doi: 10.1158/0008-5472.CAN-10-2457.

Levin SE, Zhang C, Kadlecek TA, Shokat KM, Weiss A. 2008. Inhibition of ZAP-70 kinase activity via an analog-sensitive allele blocks T cell receptor and CD28 superagonist signaling. The Journal of Biological Chemistry 283:15419-15430. doi: 10.1074/jbc.M709000200.

Lowin-Kropf B, Shapiro VS, Weiss A. 1998. Cytoskeletal polarization of T cells is regulated by an immunoreceptor tyrosine-based activation motif-dependent mechanism. Journal of Cell Biology 140:861-871. doi: 10.1083/ jcb.140.4.861.

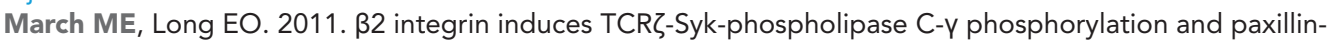
dependent granule polarization in human NK cells. Journal of Immunology 186:2998-3005. doi: 10.4049/ jimmunol.1002438.

Michel F, Grimaud L, Tuosto L, Acuto O. 1998. Fyn and ZAP-70 are required for Vav phosphorylation in T cells stimulated by antigen-presenting cells. The Journal of Biological Chemistry 273:31932-31938. doi: 10.1074/ jbc.273.48.31932.

Miletic AV, Sakata-Sogawa K, Hiroshima M, Hamann MJ, Gomez TS, Ota N, Kloeppel T, Kanagawa O, Tokunaga M, Billadeau DD, Swat W. 2006. Vav1 acidic region tyrosine 174 is required for the formation of T cell receptor-induced microclusters and is essential in T cell development and activation. The Journal of Biological Chemistry 281:38257-38265. doi: 10.1074/jbc.M608913200.

Monks CR, Freiberg BA, Kupfer H, Sciaky N, Kupfer A. 1998. Three-dimensional segregation of supramolecular activation clusters in T cells. Nature 395:82-86. doi: 10.1038/25764.

Morone N, Fujiwara T, Murase K, Kasai RS, Ike H, Yuasa S, Usukura J, Kusumi A. 2006. Three-dimensional reconstruction of the membrane skeleton at the plasma membrane interface by electron tomography. Journal of Cell Biology 174:851-862. doi: 10.1083/jcb.200606007.

Negishi I, Motoyama N, Nakayama K, Nakayama K, Senju S, Hatakeyama S, Zhang Q, Chan AC, Loh DY. 1995. Essential role for ZAP-70 in both positive and negative selection of thymocytes. Nature 376:435-438. doi: 10.1038/376435a0.

Riteau B, Barber DF, Long EO. 2003. Vav1 phosphorylation is induced by beta2 integrin engagement on natural killer cells upstream of actin cytoskeleton and lipid raft reorganization. The Journal of Experimental Medicine 198:469-474. doi: 10.1084/jem.20021995.

Robertson LK, Mireau LR, Ostergaard HL. 2005. A role for phosphatidylinositol 3-kinase in TCR-stimulated ERK activation leading to paxillin phosphorylation and CTL degranulation. Journal of Immunology 175:8138-8145.

Roumier A, Olivo-Marin JC, Arpin M, Michel F, Martin M, Mangeat P, Acuto O, Dautry-Varsat A, Alcover A. 2001. The membrane-microfilament linker ezrin is involved in the formation of the immunological synapse and in $T$ cell activation. Immunity 15:715-728. doi: 10.1016/S1074-7613(01)00225-4.

Sanderson CJ, Glauert AM. 1977. The mechanism of T cell mediated cytotoxicity. V. Morphological studies by electron microscopy. Proceedings of the Royal Society of London Series B Biological Sciences 198:315-323. doi: 10.1098/rspb.1977.0100.

Sanderson CJ, Glauert AM. 1979. The mechanism of T-cell mediated cytotoxicity. VI. T-cell projections and their role in target cell killing. Immunology 36:119-129.

Stinchcombe J, Bossi G, Griffiths GM. 2004. Linking albinism and immunity: the secrets of secretory lysosomes. Science 305:55-59. doi: 10.1126/science.1095291.

Stinchcombe JC, Barral DC, Mules EH, Booth S, Hume AN, Machesky LM, Seabra MC, Griffiths GM. 2001 a. Rab27a is required for regulated secretion in cytotoxic T lymphocytes. Journal of Cell Biology 152:825-834. doi: 10.1083/jcb.152.4.825.

Stinchcombe JC, Bossi G, Booth S, Griffiths GM. 2001b. The immunological synapse of CTL contains a secretory domain and membrane bridges. Immunity 15:751-761. doi: 10.1016/S1074-7613(01)00234-5.

Stinchcombe JC, Griffiths GM. 2007. Secretory mechanisms in cell-mediated cytotoxicity. Annual Review of Cell and Developmental Biology 23:495-517. doi: 10.1146/annurev.cellbio.23.090506.123521.

Stinchcombe JC, Majorovits E, Bossi G, Fuller S, Griffiths GM. 2006. Centrosome polarization delivers secretory granules to the immunological synapse. Nature 443:462-465. doi: 10.1038/nature05071.

Stinchcombe JC, Salio M, Cerundolo V, Pende D, Arico M, Griffiths GM. 2011. Centriole polarisation to the immunological synapse directs secretion from cytolytic cells of both the innate and adaptive immune systems. BMC Biology 9:45. doi: 10.1186/1741-7007-9-45.

Tskvitaria-Fuller I, Rozelle AL, Yin HL, Wulfing C. 2003. Regulation of sustained actin dynamics by the TCR and costimulation as a mechanism of receptor localization. Journal of Immunology 171:2287-2295. 
Tsun A, Qureshi I, Stinchcombe JC, Jenkins MR, De la Roche M, Kleczkowska J, Zamoyska R, Griffiths GM. 2011. Centrosome docking at the immunological synapse is controlled by Lck signaling. Journal of Cell Biology 192:663-674. doi: 10.1083/jcb.201008140.

Tybulewicz VL. 2005. Vav-family proteins in T-cell signalling. Current Opinion In Immunology 17:267-274. doi: 10.1016/j.coi.2005.04.003.

Ueda H, Morphew MK, Mcintosh JR, Davis MM. 2011. CD4+ T-cell synapses involve multiple distinct stages. Proceedings of the National Academy of Sciences of the United States of America 108:17099-17104. doi: 10.1073/pnas.1113703108.

Valitutti S, Dessing M, Aktories K, Gallati H, Lanzavecchia A. 1995. Sustained signaling leading to T cell activation results from prolonged T cell receptor occupancy. Role of T cell actin cytoskeleton. The Journal of Experimental Medicine 181:577-584. doi: 10.1084/jem.181.2.577.

van Oers NS, Killeen N, Weiss A. 1996. Lck regulates the tyrosine phosphorylation of the T cell receptor subunits and ZAP-70 in murine thymocytes. The Journal of Experimental Medicine 183:1053-1062. doi: 10.1084/ jem.183.3.1053.

Wernimont SA, Wiemer AJ, Bennin DA, Monkley SJ, Ludwig T, Critchley DR, Huttenlocher A. 2011. Contactdependent T cell activation and T cell stopping require talin1. Journal of Immunology 187:6256-6267. doi: 10.4049/jimmunol.1102028.

Yi J, Wu XS, Crites T, Hammer JA III. 2012. Actin retrograde flow and actomyosin II arc contraction drive receptor cluster dynamics at the immunological synapse in Jurkat T cells. Molecular Biology of the Cell 23:834-852. doi: 10.1091/mbc.E11-08-0731.

Zhao F, Cannons JL, Dutta M, Griffiths GM, Schwartzberg PL. 2012. Positive and negative signaling through SLAM receptors regulate synapse organization and thresholds of cytolysis. Immunity 36:1003-1016. doi: 10.1016/j.immuni.2012.05.017. 